\title{
Representation of galactic dynamics via isoshifts without universe expansion, dark matter and dark energy
}

\author{
Ruggero Maria Santilli \\ Thunder Fusion Corporation, Tarpon Springs, U.S.A
}

Email address:

research@thunder-energies.com

To cite this article:

Ruggero Maria Santilli. Representation of Galactic Dynamics Via Isoshifts Without Universe Expansion, Dark Matter and Dark Energy. American Journal of Modern Physics. Vol. 4, No. 2, 2015, pp. 26-43. doi: 10.11648/j.ajmp.20150402.11

\begin{abstract}
In this paper, we outline mathematical, theoretical and experimental studies by the author on the anomalous redshifts (blueshifts) of light passing through cold (hot) gases without relative motion, that are known as Santilli isoredshifts (isoblueshifts); we review the rather vast experimental and visual evidence on the lack of expansion of the universe and related cosmological conjectures, including the lack of acceleration, big bang and dark energy; we recall a number of insufficiencies of the conjecture of dark matter, with particular reference to its lack of quantitative representation of anomalous redshifts of galactic stars; we then present, apparently for the first time, a quantitative and time invariant representation of the anomalous redshift of galactic stars via Santilli isoredshifts and isoblueshifts without dark matter by merely admitting the astrophysical evidence that galaxies are indeed filled up with an actual, physical, detectable, gaseous medium causing deviations from the Doppler shift law; and we point out that the emerging new vistas of the universe do preserve Einstein's axioms of special relativity and merely require broader realizations.
\end{abstract}

Keywords: Dark Matter, Spacial Relativity, Isorelativity

\section{Problematic Aspects of the Dark Matter Conjecture}

As it is well known, the sole experimental information we receive from far away galaxies is their light. All other numerical values existing in contemporary astrophysics and cosmology are derived from experimental data on light via theoretical assumptions not directly verifiable on Earth.

More specifically, the sole experimental information we have on far away galaxies is Hubble's law [1] on the cosmological redshift of galaxies, hereon indicated with the symbol $z_{g}$, according to which galactic light is redshifted in a way proportionate to their distance $d_{g}$ from Earth

$$
z_{g}=\frac{\omega_{e}}{\omega_{o}}-1 \approx H d_{g}
$$

where $H$ is Hubble's constant, $\omega_{o}$ is the frequency of light at the origin, and $\omega_{e}$ is the light frequency measured on Earth.

As it is equally well known, inter-galactic space is far from being empty, because it is filled up with a medium, hereon called inter-galactic medium, composed by electromagnetic waves originating from all stars in the universe, Hydrogen and other gases, elementary particles and other physical entities.

For the generally unspoken intent to reconcile Hubble's law (1.1) with special relativity [2-5], the inter-galactic space has been assumed as being empty, thus allowing the representation of the cosmological redshift with the conjecture of the expansion of the universe (see Ref. [4] for a general review on the expansion of the universe).

According to this conjecture, galaxies are assumed move away from us at a speed, hereon denoted $v_{g}$, in which case Hubble's law (1.1) is interpreted via the Doppler axiom of special relativity (see, e.g., Ref. [7] and vast literature quoted therein)

$$
z_{g}=\frac{\omega_{e}}{\omega_{o}}-1 \approx H d_{g} \approx \frac{v_{g}}{c} .
$$

The above interpretation was based on the following generally tacit assumptions:

ASSUMPTION 1.1: The inter-galactic space is exactly represented by the Minkowski spacetime [4] $M(x, \eta, I)$ with coordinates, metric, unit and invariant 


$$
\begin{gathered}
x=\left(x_{\mu}\right)=\left(x^{1}, x^{2}, x^{3}, t\right), \\
\eta=\text { Diag. }\left(1,1,1,-c^{2}\right), \\
I=\text { Diag. }(1,1,1,1), \\
x^{2}=x^{\mu} \eta_{\mu v} x^{v}=x_{1}^{2}+x_{2}^{2}+x_{3}^{2}-t^{1} c^{2} ;
\end{gathered}
$$

ASSUMPTION 1.2: The Lorentz symmetry [2] is the universal symmetry for the large scale structure of the universe (namely, for distances under which galaxies can be assumed as being massive points)

$$
\begin{gathered}
x^{1^{\prime}}=x^{1}, x^{2^{\prime}}=x^{2}, \\
x^{3^{\prime}}=\gamma\left(x^{3}-\beta x^{4}\right), \\
x^{4^{\prime}}=\gamma\left(x^{4}-\beta x^{3}\right), \\
\beta_{g}=\frac{v_{g}}{c}, \gamma=1 / \sqrt{1-\beta_{g}^{2}} ;
\end{gathered}
$$

ASSUMPTION 1.3; The cosmological redshift is exactly described by the Doppler axiom of special relativity [7]

$$
\omega_{e}=\omega_{o} \gamma\left[1 \pm \frac{v_{g}}{c} \cos \alpha\right],
$$

that, for a point like distant source with angle of aberration $\alpha=90^{\circ}$, reduces to the expression in first order expansion in terms of $\beta_{g}=v_{g} / c$

$$
\omega_{e}=\omega_{o}\left(1 \pm \frac{v_{g}}{c}\right)
$$

from which assumption (1.2) follows, where redshift represented with the sign " $-"$ and blueshift is represented with the sign " + ."

According to the above assumptions, all stars belonging to a given galaxy should have the same cosmological redshift because distances between stars of the same galaxy, hereon called inner-galactic distances and denoted $d_{s}$, are ignorable with respect to the distance $d_{g}$ of the considered galaxies from us.

Doubts on the latter expectation began to emerge in the 1930s (see the historical notes of Ref. [7]). In 1970, the astrophysicists Vera Rubin and Kent W. Ford [8]measured for the first time the cosmological redshift of individual stars belonging to a given galaxy, and reported anomalies over assumption (1.2) depicted in Fig. 1.

As one can see, the indicated deviations are expressed in terms of the distance of stars from the galactic center and their rotational speed

$$
v_{s}=\frac{g M}{r_{s}^{2}}
$$

where $g$ is the gravitational constant, $M$ is the mass of the galaxy enclosed by the orbital motion, and $r_{s}$ is the average radius of the orbit.

In this case, the measured total redshift of individual stars belonging to a given galaxy can be characterized by the total redshift characterized by two contributions, the redshift $z_{g}$ due to the galaxy as a whole, and the deviation $z_{s}$ of the redshift of individual stars from $z_{g}$

$$
z=z_{g}+z_{s}
$$

In an attempt to reconcile the anomalies of Fig. 1 with Newtonian, Galilean and Einsteinian theories, a rather wide consensus was reached on the assumption that the anomalous behavior of individual stars of a given galaxy is due to an invisible and undetectable substance called dark matter which is filling up all galaxies (see historical account [8], representative references [9-17] and review [18] with vast additional literature quoted therein)
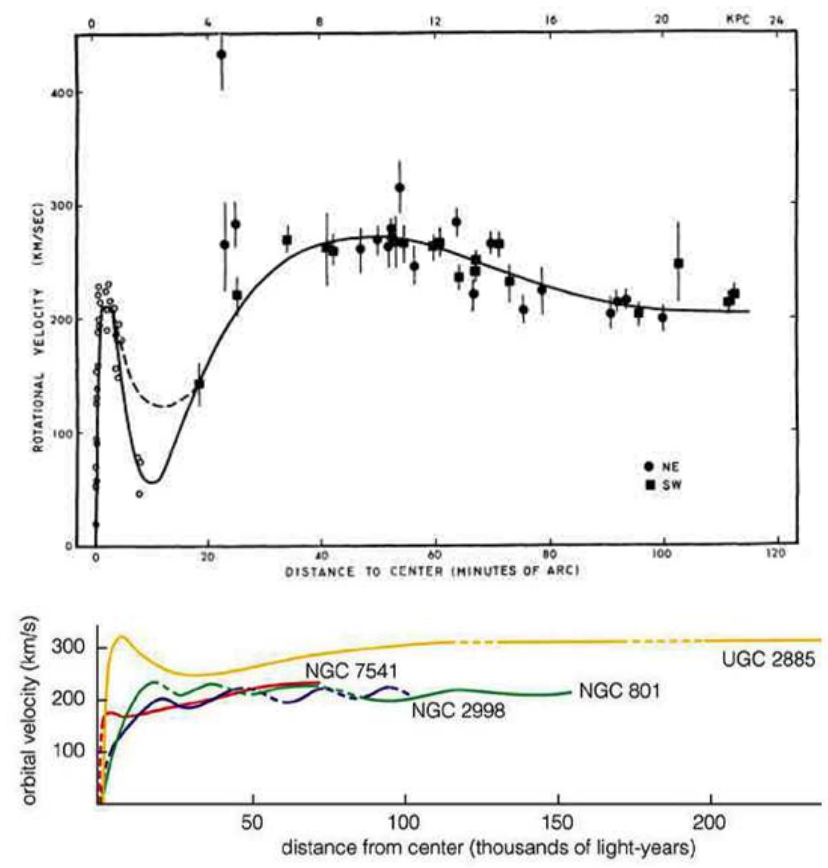

Figure 1. Views of various anomalous redshifts of galactic stars, the top view from Ref. [8] and the bottom view from various sources from refs. [9-18]. Note the referral of the data to the orbital rotations of the stars of a given galaxy. It should be stressed that this rotation motion is conjectural because based on Assumptions 1.1, 1.2 and 1.3 and that the sole measured quantity is the anomalous redshift of individual stars of a given galaxy compared to the cosmological redshift of the galaxy as a whole.

Despite vast efforts [6-18] over decades, the conjecture of dark matter remains afflicted by a number of insufficiencies whose identification is evidently a necessary pre-requisite for basic advances in the field.

Firstly, we should indicate hat, contrary to a rather popular belief in astrophysics (but not in acoustics), the rotation of galactic stars in a plane perpendicular to $d_{g}$ provides no measurable contribution to the anomalous redshift of Fig. 1.

Consider first the nonrelativistic case of a galaxy with its 
symmetry plane perpendicular to $d_{g}$ and with $d_{s} \ll d_{g}$. Remember that, both in electromagnetism and acoustics, the Doppler effects can only occur under a variation of the distance from the observer. It is evident that we have no appreciable variation of distance for the case of far away galaxies whose symmetry place is perpendicular to $d_{g}$ (top view of Fig. 2) and, consequently, under such a configuration, there cannot be any Doppler effect due to the rotational motion of stars.

By contrast, actual measurements (those of the redshift) indicate that the biggest deviations from law (1.2) occur in the vicinity of galactic center where rotations can have considerable orbital speeds.

Consider then the case of a galaxy with the galactic plane at an angle with respect to $d_{g}$ (bottom view of Fig. 2). In this case, we do have a variation of the distance $d_{s}$ and, therefore, we do have Doppler effects, but two opposite effects, one for stars on one side of the galactic center and the opposite effect for stars in the other side of the galactic center evidently due to opposite rotational speeds with respect to Earth.

However, experimental data on the redshift of individual stars dismiss these opposite Doppler contributions, again, because inspection of the data of Fig. 1 establishes that the redshift anomalies are the same for both sides of the galactic

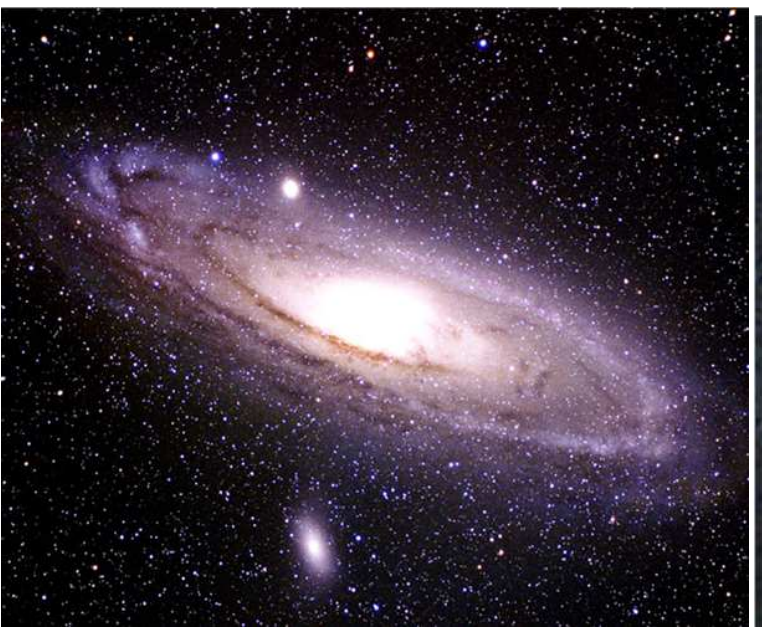

Figure 2. Pictures of representative galaxies from NASA archives, the left view representing a galaxy with its symmetry plane perpendicular to the distance from Earth, while the right view depicts a galaxy with its symmetry plane at an angle to said distance.

But again, $v_{s} / c \approx 0$. Therefore, we confirm the indicated lack of appreciable relativistic contributions to the anomalous redshift because

$$
\omega_{e} \approx \omega_{o}
$$

Once theoretical and experimental evidence eliminates measurable contributions to anomalous redshift of galactic star due to their rotations, the conjecture of dark matter emerges as the only possibility to maintain the validity of special relativity for galactic dynamics.

Unfortunately, the dark matter conjecture is itself afflicted by serious insufficiencies treated in the refereed literature (see later on Refs. [20-59], such as:

1.a. The dark matter conjecture has not provided a numerical

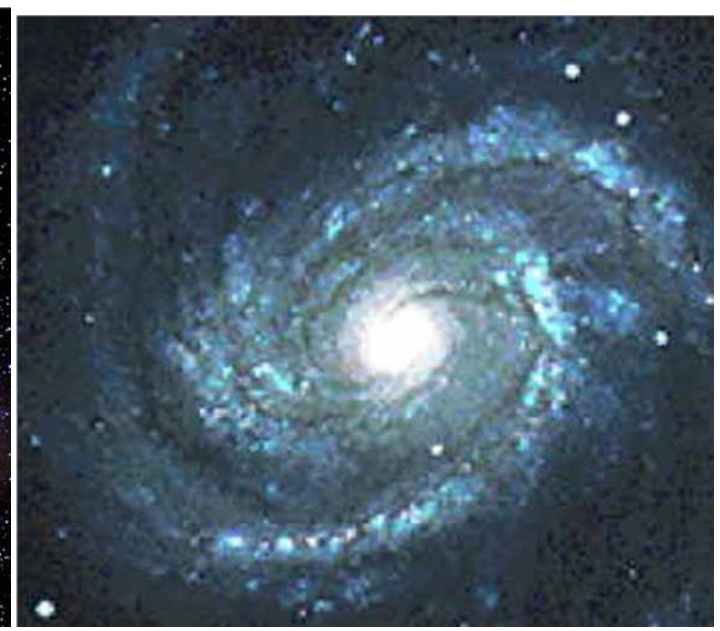

center.

Additionally, the Doppler effects due to rotation of stars at an angle with respect to $d_{g}$ cannot provide any measurable contribution to the anomalies of Fig. 1 because speeds of stars are measured in kilometer per house, while the speed of light is 300, 000 kilometer per seconds, resulting in an essentially null value of $v_{s} / c$ with an essentially null Doppler shift of light frequency.

For the relativistic case, consider Doppler's law (1.5) for the aberration angle $\alpha=90^{\circ}$,

$$
\omega^{\prime}=\omega_{0} \gamma_{s}
$$

But

$$
\gamma_{s} \approx 1 \pm \frac{v_{s}}{c}
$$

Therefore, Eq. (1.5) becomes

$$
\omega_{e}=\omega_{o} \gamma \approx \omega_{o} \pm \omega_{o} \frac{v_{s}}{c} .
$$

representation of the anomalous redshifts of Fig. 1, and none is expected under the assumption that dark matter is uniformly distributed in a galaxy, evidently because in this case all possible effects on individual stars cancel out.

1.b. In order to achieve a meaningful effect, dark matter has to be distributed adhoc with a given density in front of a given star, but then the dynamics of the remaining stars is evidently disproved by experimental evidence.

1.c. Newton's require that, in the event galaxies are filled up by dark matter, they should contract, contrary to clear experimental evidence that galaxies do not contract, on the contrary, experimental evidence establishes that galaxies under formation expand. 
1.d. The value of the rotational speeds of individual galactic stars theoretically predicted by the dark matter conjecture imply internal changes in the distribution of galaxies depending on their distance to their center, which changes appear to be disproved by about one century of astrophysical records.

1.e. In order for rotations to produce any measurable frequency shift, their rotational speed should be relativistic, in which case individual stars would no longer be visible within a galaxy, and no galaxy could possibly be stable in view of the virial theorem (see Section 4.3).

For the case of the expansion of the universe, the general trend has been that of introducing new conjectures in support of a preceding insufficient conjecture (see Section 3). all conjectures being aimed at the generally unspoken intent of maintaining special relativity for the larger scale structure of the universe.

There is a similar trend is under way for galactic dynamics. In fact, following insufficiencies 1.A, 1.B, 1.C and others, a general trend is that of maintaining the validity of special relativity via the introduction of new conjectures, such as: the hypothetical neutralino, the sterile neutrino, the WIMPS (new, hypothetical, weakly INTERACTING massive particles not detected in particle physics laboratories), and other conjectures that, generally by conception, are not experimentally verifiable on Earth.

The view presented in this paper is that, while conjectures aimed at maintaining special relativity for galactic dynamics should continue to be studied, time has arrived for the astrophysics community to jointly address the experimental evidence achieved on Earth according to which the anomalous redshifts of Fig. 1 are due to deviation from special relativity caused by the inner-galactic medium.

\section{Isoredshifts and Isoblueshifts}

As it is well known, the 20th century realization of special relativity (herein referred to as special relativity [2]) is exactly valid under the conditions originally conceived by Einstein and experimentally verified beyond doubt, namely, for: 2.A) point particles and electromagnetic waves; 2.B) propagating in empty space; $2 . C$ ) when considered from inertial reference systems.

Santilli's main contention studied in a variety of works over decades [20-59] is that special relativity is "inapplicable" (rather than "violated") under sufficient deviations from Einstein's original conditions 2.A, 2.B, and 2.C.The deviations from special relativity studied in this paper are those for dynamics within generally inhomogeneous and anisotropic physical media because, in this case [loc./cit.]: 1.A*)particles cannot be consistently abstracted as massive points, thus requiring the representation of extended and deformable particles; 2. $\mathrm{B}^{*}$ ) the inhomogeneity and anisotropy of physical media cannot be consistently represented via the Minkowskian geometry; and 2.C*) there is no possibility of introducing inertial reference systems within physical media due to then notorious resistance that, contrary to a rather general belief, cannot be consistently eliminated by reducing extended particles to massive points and electromagnetic waves to photons. .

In order to confront expected deviations of special relativity with physical reality, beginning with studies at the Department of Mathematics of Harvard University in the late 1970s, Santilli initiated the construction of a new mathematics for the treatment of dynamics within physical media, today known as Santilli IsoMathematics, where the prefix "iso" is intended to indicate the preservation of the original axioms.

The first formulation of isomathematics was presented in volumes [20] (written at Harvard University following the delivering of a seminar course in the field) and was based on the lifting of the conventional associative product $A B$ between generic quantities $A, B$ (functions, matrices, operators, etc.) into the associativity-preserving form called isoproduct

$$
A B \rightarrow A \hat{\times} B=A \hat{T} B
$$

where the quantity $\hat{T}$, called the isotopic element, is positive-definite, but otherwise possesses an unrestricted functional dependence on all needed local variables, such as time $t$, coordinates $r$, velocities $v$, density $\rho$, temperature $\tau$, frequency $\omega$, etc.

The realization of the isotopic elements we use for astrophysical studies is given by (see, later on, Refs. [23] for other realizations)

$$
\begin{aligned}
& \hat{T}=\operatorname{Diag} \cdot\left(\frac{1}{n_{1}^{2}}, \frac{1}{n_{2}^{2}}, \frac{1}{n_{3}^{2}}, \frac{1}{n_{4}^{2}}\right), \\
& n_{\mu}=n_{\mu}(t, r, v, \rho, \tau, \omega, \ldots)>0, \\
& \mu=1,2,3,4,
\end{aligned}
$$

where the quantities $n_{v}$, called the characteristic quantities of the medium considered.

It should be stressed that, rather then being "free parameters," the characteristic quantities are instead

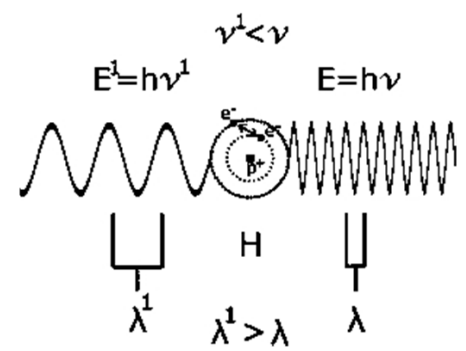

Figure 3. This diagram illustrates the essentially quantum mechanical origin of Santilli isoredshifts and isoblueshifts (jointly called isoshifts). Isoredshifts are illustrated from right to left via an electromagnetic wave hitting an atom in its ground state, which is excited in the process, while the electromagnetic wave loses energy with consequential redshift. Isoblueshifts are illustrated from left to right and deal with the inverse process in which an atom in its excited state is hit by an electromagnetic wave, by returning to the ground state, while the electromagnetic wave acquires energy and experiences a blueshift. 
measurable quantities as we shall see. In fact, $n_{4}$ is the familiar index of refraction intended as a geometrization of the density with normalization to the value $n_{4}=1$ for the vacuum, and the $n_{k}, k=1,2,3$, represent the inhomogeneity (e.g., the variation of the density, as it is the case for Earth's atmosphere), the anisotropy (e.g., the anisotropy caused by rotations, as it is also the case for Earth's atmosphere).

Isomathematics emerged via the systematic lifting of all aspects of 20th century applied mathematics via product (2.1), including the isotopies of the various branches of Lie's theory first presented in Refs. [20], today known as the Lie-Santilli isotheory, with isoalgebra and isogroup for Hermitean generators

$$
\begin{gathered}
{\left[J_{m} \hat{\jmath} J_{n}\right]=J_{m} \hat{\times} J_{n}-J_{n} \hat{\times} J_{m}=J_{m} \hat{T} J_{n}-J_{n} \hat{T} J_{m}=C_{m . n}^{k} J_{k}} \\
A(w)=e^{H \hat{T} w i} A(0) e^{-i w \hat{T} H}
\end{gathered}
$$

where the isogroup has been restricted, for simplicity to the one-dimensional case in the parameter $w$

Subsequently, consistency conditions with the number theory required the isotopies of ordinary numbers $n$ (real, complex and quaternionic numbers). These isotopies were achieved for the first time in memoir [21] of 1993 by assuming for basic, multiplicative, right and left unit the inverse of the isotopic element, $\hat{I}=1 / \hat{T}>0$, today known as Santilli isounit, resulting in numbers $\hat{n}=n \hat{I}$, today known as isonumbers, and their sets are known as isofields.

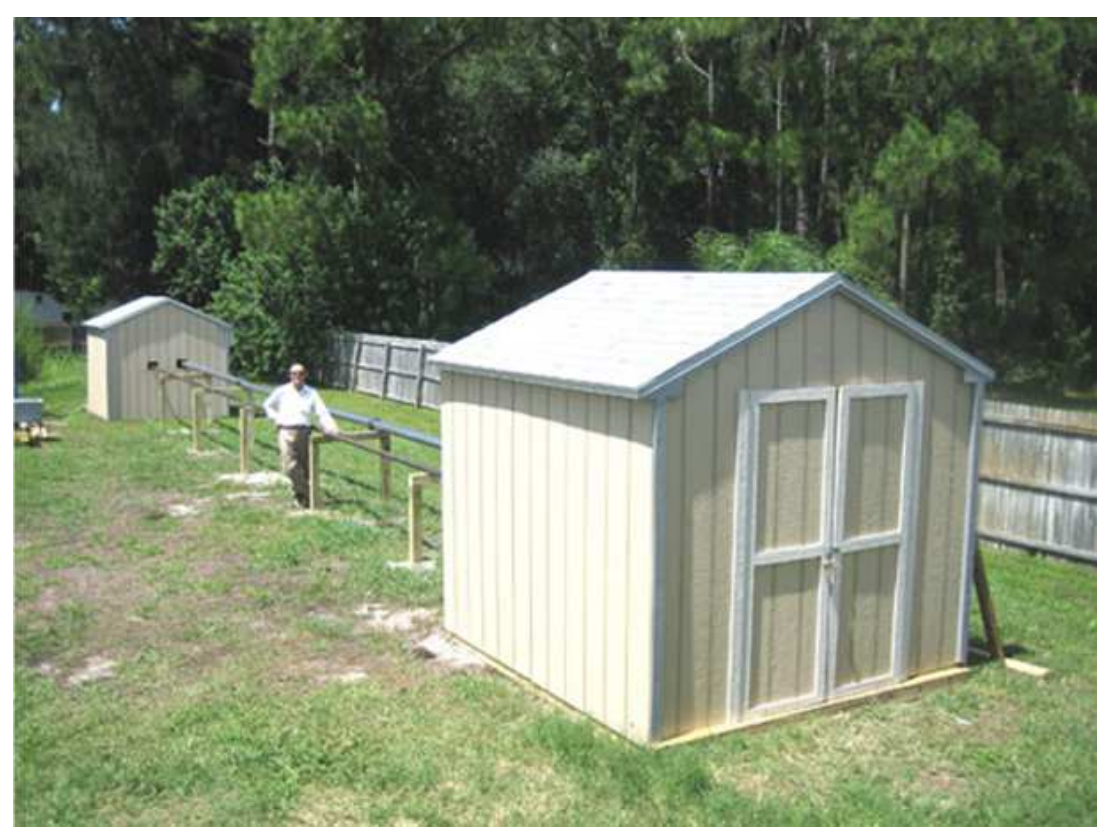

Figure 4. A view of the IsoShift Testing Station built by Santilli in 2009 including: an air conditioned cabin right containing a blue laser light; an air conditioned station in the left containing wavelength analyzers; the laser light and the detectors being interconnected by a steel tube 60 'long containing air at pressure; and means to increase or decrease the temperature of the steel tube.

The entire original formulation of isomathematics [20] was then lifted in such a way to admit Santilli isounit as the basic left and right unit at all levels. In particular, all numerical values have to be isoscalars, as a result of which coordinates assume the isotopic form $\hat{r}=r \hat{I}_{r}$, velocities have the form $\hat{v}=v \hat{I}_{v}$, etc.

The achievement of time invariance (the prediction of the same numerical values under the same conditions at different times) required the completion of isomathematics with the isotopies of Newton's differential calculus, first presented in Ref. [22] of 1996, with Santilli isodifferential and isoderivative

$$
\begin{gathered}
\hat{d} \hat{r}=\hat{T} d \hat{r}=\hat{T} d[r \hat{I}(t, r, v, \rho, \tau, \omega, \ldots)]=d r+\hat{T} d \hat{I}, \\
\frac{\hat{\partial} \hat{F}(\hat{r})}{\hat{\partial} \hat{r}}=\hat{I} \frac{\partial \hat{F}(\hat{r})}{\partial \hat{r}},
\end{gathered}
$$

$$
\begin{gathered}
\hat{I}=1 / \hat{T}=\hat{I}(t, r, v, \rho, \tau, \omega, \ldots)>0, \\
\hat{I} \hat{\times} A=A \hat{\times} \hat{I}=A .
\end{gathered}
$$

In turn, the achievement of the novel isodifferential calculus permitted Santilli to formulate the first known axiomatically consistent extension of Newton's equations, from their historical characterization of massive points, to extended, generally non-spherical and deformable bodies under the most general known linear and non-linear, non-local and non-local, Hamiltonian and non-Hamiltonian forces. These generalized equations were first presented in memoir [22] of 1996, are today known as the Newton-Santilli isoequations, and can be written in the form

$$
\hat{m} \hat{\times} \frac{\hat{d} \hat{r}}{\hat{d} \hat{t}}=\hat{F}^{S A}(\hat{t}, \hat{r}, \hat{v}, \hat{a}, \ldots),
$$

where: all scalar quantities are elements of Santilli isofields, 
and $S A$ ( NSA ) stands for variational selfadjointness (non-selfadjointness), namely, the verification (violation) of the integrability conditions to admit a conventional Hamiltonian) [20].

As one can see, the most important meaning of Eqs. (2.5) is that of turning highly non-Hamiltonian equations on conventional spaces over conventional fields into a fully Hamiltonian form on isospaces over isofields. This mean objective is achieved via the embedding of all non-linear, non-local and non-Hamiltonian forces into Santilli isodifferentials and isoderivatives. appearance In turn, the recovering of $S A$ permitted the achievement, for the first time in Ref. [22] of a variational principal NSA systems which, in turn, permitted the the first known unique and unambiguous map of NSA interactions into a covering of quantum mechanics known under the name of hadronic mechanics, first proposed in Ref. [20] (see page 112, Volume 2 of Ref. ]20 for the first appearance of the name, memoir [22] for mathematically consistent formulations, and Refs. [23 for a comprehensive treatment).

Nowadays, Santilli isomathematics is referred to the lifting of the entire 20th century applied mathematics in such a form to admit $\hat{I}$ as the correct left and right un at all possible levels, thus including the number theory, functional analysis, differential calculus, algebras, groups, symmetries, geometries, topologies, etc. [23]:

Readers may be interested in knowing the generalization of isomathematics known as Santilli Lie-admissible genomathematics, also introduced for the first time in monographs [20] (see the recent presentation [24]) specifically constructed for time irreversible events.

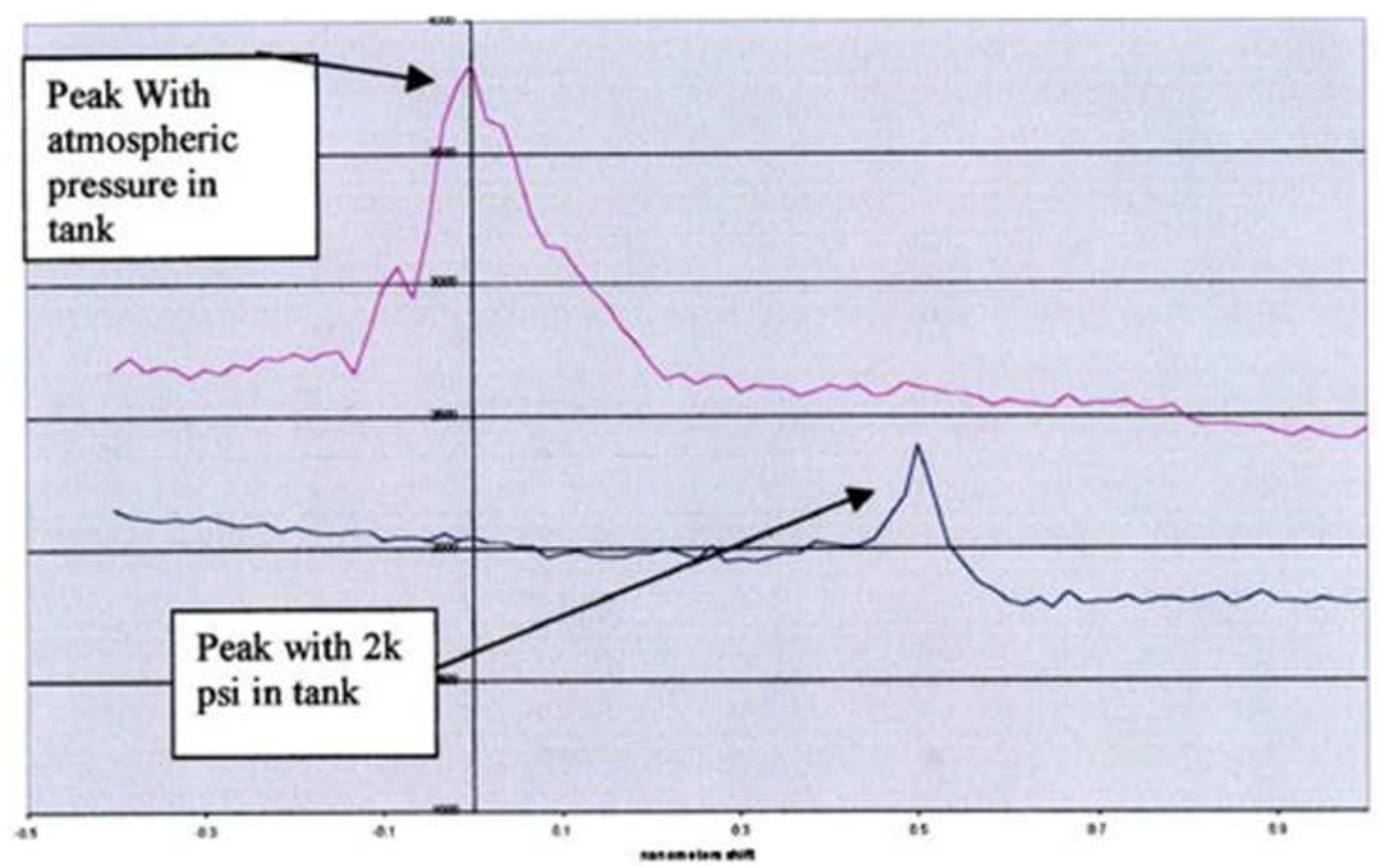

Figure 5. The first experimental evidence on the existence of Santilli IsoRedShift obtained on the IsoShift station of Fig. 3 [37].

Readers may also be interested in the generalization of genomathematics into the irreversible hypermathematics $[22,23,25]$ which allows multi-valued (rather than multi-dimensional) cosmological models, such as a $(3+1)$ dimensional space-time containing four coexisting but different universes given by matter and antimatter universes, plus Ying's twin universes [26].

On physical grounds, isomathematics requires the lifting of the Minkowski space-time [4] into the Minkowski-Santilli iso-space-time $\hat{M}(\hat{x}, \hat{\eta}, \hat{I} \mathrm{~m}$ where $\hat{x}=x \hat{I}$ and $\hat{\eta}=\hat{T} \eta \mathrm{m}$ with isoinvariant first achieved in Ref. [27] of 1983

$$
\hat{x}^{\hat{2}}=\hat{x}^{\mu} \hat{\times} \hat{\eta}_{\mu v} \hat{\times} \hat{x}^{\nu}=\left[x^{\mu}\left(\hat{T}_{\mu}^{\theta} \eta_{\theta v}\right) x^{\nu}\right] \hat{I}=\left(\frac{x_{1}^{2}}{n_{1}^{2}}+\frac{x_{2}^{2}}{n_{2}^{2}}+\frac{x_{3}^{2}}{n_{3}^{2}}-t^{2} \frac{c^{2}}{n_{4}^{2}}\right) \hat{I} .
$$

The use of the Lie-Santilli isotheory, Eqs, (2.3), for the isotopic element (2.3) then allowed the construction the isotopies of the Lorentz symmetry [2] resulting in the universal symmetry of isoinvariant $(2,6)$, also introduced for the first time in Ref. [27] at the classical level and Ref. [28] at the operator level. the new isosymmetry is today known as the Lorentz-Santilli isosymmetry and can be written in the form

$$
\begin{gathered}
x^{11}=x^{1}, x^{.2}=x^{2}, \\
x^{3}=\hat{\gamma}\left(x^{3}-\hat{\beta} \frac{n_{3}}{n_{4}} x^{4}\right),
\end{gathered}
$$




$$
\begin{gathered}
x^{4}=\hat{\gamma}\left(x^{4}-\hat{\beta} \frac{n_{4}}{n_{3}} x^{3}\right), \\
\hat{\beta}=\frac{v_{3} / n_{3}}{c_{o} / n_{4}}, \hat{\gamma}=\frac{1}{\sqrt{1-\hat{\beta}^{2}}} .
\end{gathered}
$$

Santilli then conducted systematic studies of the main aspects of the Poincaré symmetry [3], including monographs [29] of 1991, with the isotopies of: the rotational symmetry [30]; the pin symmetry [31]; the Poincaré symmetry [32] including the universal symmetry of all infinitely possible Riemannian line elements that are a particular case of invariant (2.6); the spinorial covering of the Poincaré symmetry [33] and the Minkowskian geometry [34]; and the nuclear isospin symmetry and local realism [35] (see monographs [23] for a comprehensive review).

The "direct universality" (namely, the invariance of all infinitely possible symmetric and non-singular line elements in $(3+1)$ spaces directly in the frame of the experimenter) was proved in Ref. [36] (see, later on,m independent verifications $[45,46])$, thus including the invariance of all; infinitely possible Minkowskian, Riemannian, Fynslerian and other line elements.

The axiom-preserving lifting of special relativity permitted by isomathematics for the study of dynamics within physical media was first identified in Refs. [29], then treated in Refs. $[23,44]$, and it is known nowadays as isorelativity. In this paper we present experimental verifications for light propagating within gaseous media. Refs. [44] (see also the independent review [57]) present experimental verifications for various additional interior dynamical conditions ins. A recent presentation of isorelativity and its experimental verifications is available in lectures [65].

A consequence of the Lorentz-Santilli isosymmetry particularly important for this paper is the uniquely and unambiguously lifting of Doppler's law (1.5) into the Doppler-Santilli isolaw [27] (see the first general treatment in Refs. [29])

$$
\omega_{e}=\omega_{o} \hat{\gamma}\left[1 \pm \frac{v}{c} \frac{n_{4}}{n_{v}} \cos \alpha\right]
$$

where we have ignored isotrgonometric functions for simplicity [23], and $n_{v}$ is the space characteristic quantity in the $v$-directions.

Readers should keep in mind that we are dealing with the geometrization and the invariance of generally inhomogeneous and anisotropy media for which the numerical values of the Doppler-Santilli isolaw are generally different for different directions.

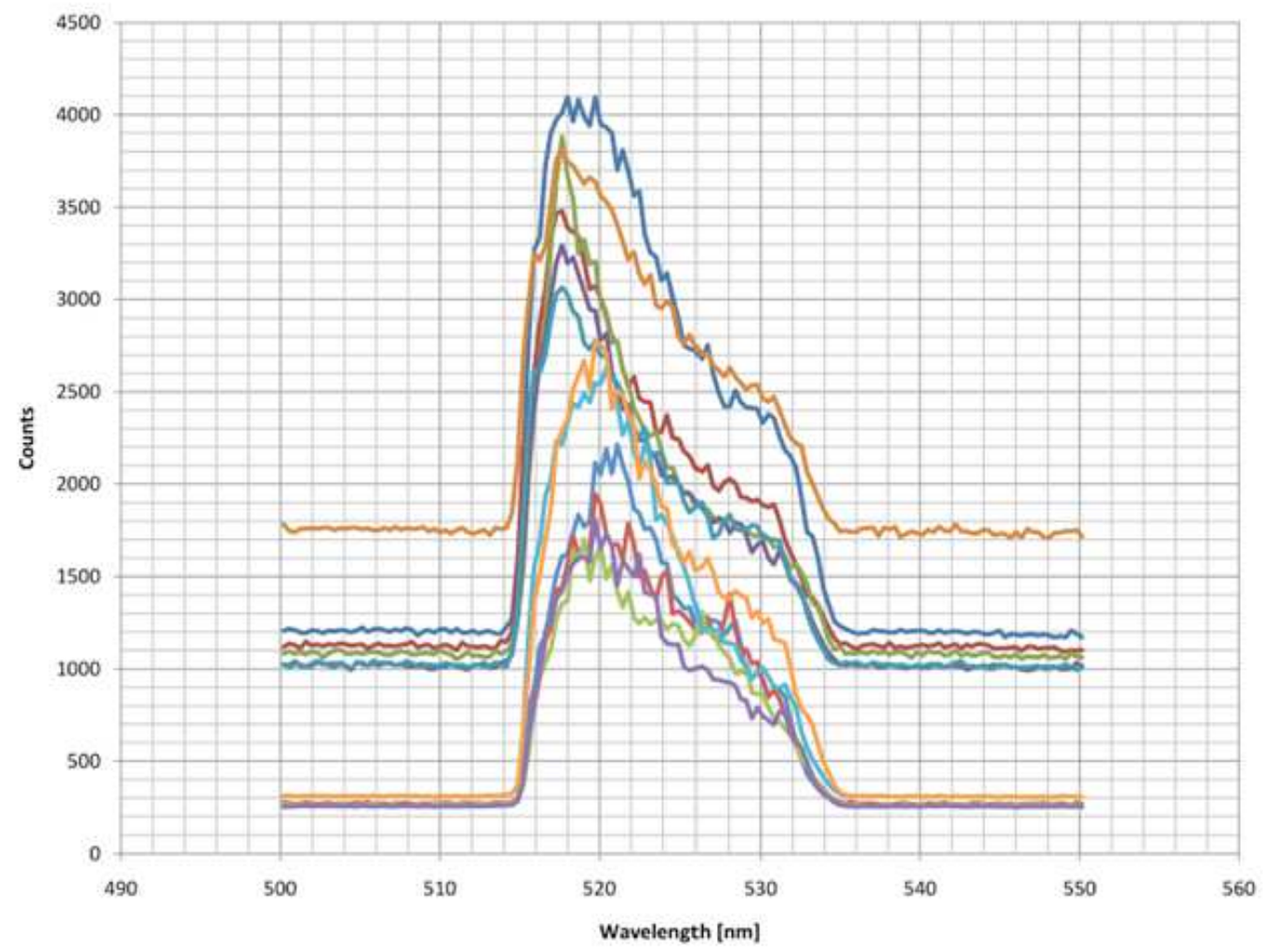

Figure 6. The first experimental evidence on the increase of the IsoRedShift with the pressure or, equivalently, with the increase of the traveled distance of light, obtained on the IsoShift station of Fig. 33.

Isolaw $(2,8)$ can be written in first order expansion

$$
\omega_{e} \approx \omega_{o}\left(1 \pm \frac{v}{c} \frac{n_{4}}{n_{v}}+\ldots\right)
$$


By recalling the arbitrary functional dependence of the characteristic quantities, we have the expansion in the distance $d$ traveled by light

$$
\frac{n_{4}}{n_{v}}=1 \pm K(t, r, v, \rho, \tau, \omega, \ldots) d,
$$

under which isotopic law (2.8) can be written in first expansion

$$
\omega_{e}=\omega_{0}\left(1 \pm \frac{v}{c} \pm F(t, r, v, \rho, \tau, \omega, \ldots) d+\ldots\right),
$$

where

$$
F=\left[\frac{v^{2}}{c^{2}} \frac{n_{4}}{n_{3}}\right]_{v=0} \neq 0,
$$

Examination of the above isolaw led Santilli to formulate in Refs. [29] of 1991 the hypothesis of isoredshift (isoblueshift) which are given by a frequency shift for light propagating within physical media at low temperature (at high temperature) given by loss of energy (acquisition of energy) by light to (from) the physical medium without any relative motion between the source, the medium and the observer according to the isoshift law

$$
\omega_{e}=\omega_{0}(1 \pm F(t, r, v, \rho, \tau, \omega, \ldots) d+\ldots) .
$$

In the above isolaw, the function $F$ is essential to represent local variations of the physical characteristics of the medium along the trajectory of light over the distance $d$.

An illustration is given by Sunlight at the horizon for which $d \approx 6,000 m$, along which density and temperature begin with values at sea level to reach rarefied and low temperature conditions in the upper layers of the atmosphere.

One should keep in mind the invariance over time of isotopic law (2.13) because it is uniquely and unambiguously derived from the Lorentz-Santilli isosymmetry (2.7) for the particular case in which Doppler's term $v / c$ is ignorable with respect to the remaining terms.

By recalling that scattering is a molecular effect, Santilli isoredshift and isoblueshift and IBR are effects occurring at the atomic level, as illustrated in Fig. 3.

The first experimental verification of IRS and isoredshift was provided by Santilli in 2010 [37] and then confirmed by independent scholars in Refs. [35-42] (see Figs. 4-7). These tests were conducted for a blue laser light propagating within a gaseous medium at temperatures above $120^{\circ} \mathrm{F}$ and below $80^{\circ} \mathrm{F}$, respectively.

Systematic measurements were then conducted by Santilli and his associates [39] for direct Sunlight, thus with the exclusion of contributions from the atmosphere surrounding the Sun. In this way, Santilli established that in the transition from the Zenith to the horizon, Sunlight experiences a redshift of the entire visible spectrum of about $100 \mathrm{~nm}$. These measurements were then independently confirmed at Sunsets and Sunrise both in the U.S.A. and Europe [41-43].
Since there is no appreciable relative motion between the center of gravity of Earth and Sun, the above redshift is a Santilli isoredshift. Note that there is a tangential motion of the observer due to Earth;s rotation which is away from the Sun at Sunset and toward the sub at Sunrise.

However, simple calculations show that the conventional Doppler contribution due to Earth rotation is of the order of $10^{-6} \mathrm{~nm}$, thus being completely ignorable in full agreement with the transition from the Doppler-Santilli isolaw (3.12) to Santilli isolaw (2.13).

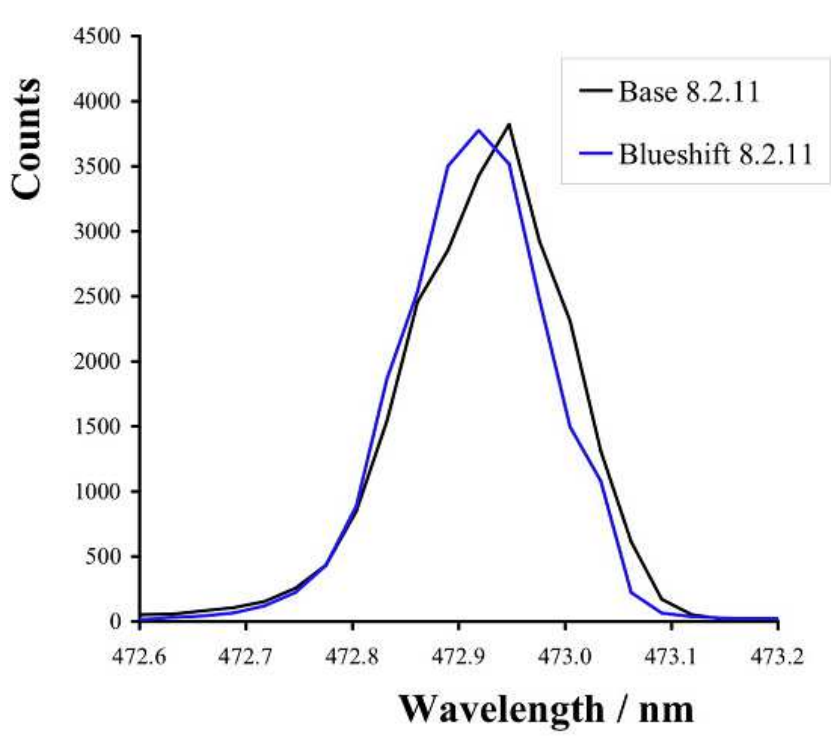

Figure 7. The first experimental evidence on the existence of Santilli isoblueshifts obtained on the IsoShift station of Fig. 3 via the cooling of the steel tube below $80^{\circ}$ R. [37].

A few comments are now in order. Firstly, we should note that, under the Lorentz-Santilli isosymmetry, the speed of light within (transparent) physical media is a local variable with value

$$
C=\frac{c}{n_{4}(t, x, v, \rho, \tau, \omega, \ldots)}
$$

On historical grounds, it should be recalled that Lorentz [2] first attempted to achieve the symmetry for the above locally varying speed of light, but had to restrict his historical symmetry to the case of a constant speed $c$ due to insurmountable technical difficulties.

Santilli did achieve in Ref. [27] the solution of the historical Lorentz problems (referred to the invariance of locally varying speeds of light), nut only after a decade of preparatory studies on the isotopies of Lie's theory.

Secondly, we should note on grounds of scientific ethics that Santilli isoredshifts and isoblueshifts depart from special relativity on various grounds. As it is well known, the speed of light is the central invariant of special relativity, while light assumes locally variable characteristics when propagating within physical media according to isosymmetry (2.7).

Thirdly, we should note that Santilli isoredshifts and isoblueshifts are structurally irreversible over time by conception and experimental verification, evidently due to the 
variation of energy $E=\bar{h} \omega$.
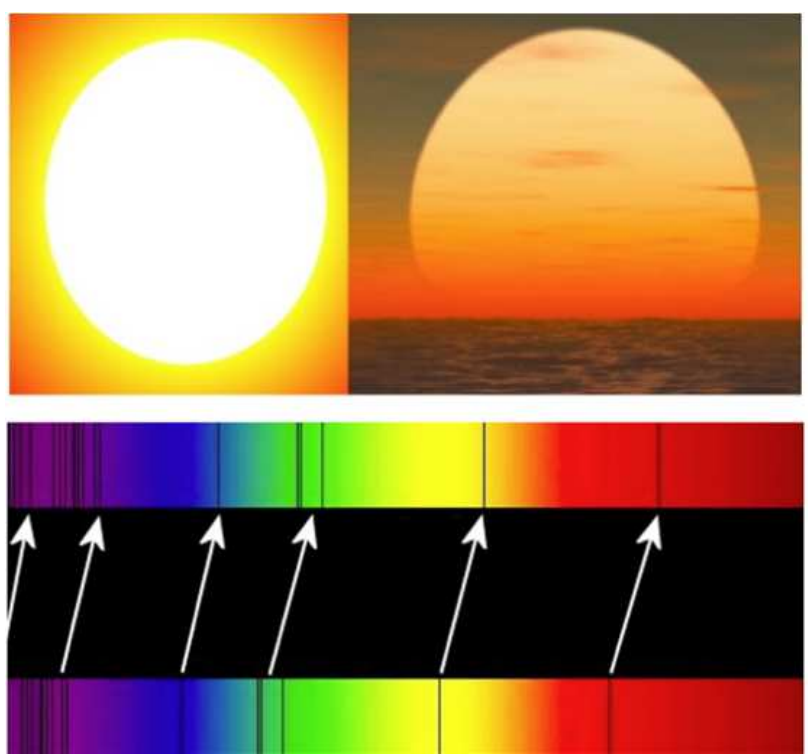

Figure 8. Beginning with monographs [29] of 1991, Santilli's mathematical, theoretical and experimental studies on the IsoRedShift were aimed at showing that the redness of the Sun at Sunset and Sunrise is precisely a redshift due to loss of energy by light to our atmosphere, thus departing from special relativity within a physical medium (only). The numerous alternative interpretations aimed at maintaining special relativity for the redness of the Sun at the horizon have been dismissed in refereed papers, such as Refs. [37-53].

On rigorous grounds, this irreversible character would require a treatment via the Lie-admissible genomathematics $[23,24]$. To avoid excessive mathematical treatments, in this paper we have represented irreversibility of the iso-space-time via a non-invariant time dependence of the isounit of the type

$$
\hat{I}(t, \ldots .)=\hat{I}^{\dagger}(t, \ldots) \neq \hat{T}(-t, \ldots)=\hat{T}^{\dagger}(-t, \ldots)=\hat{T}^{\dagger}(-t, \ldots .) \text {. }
$$

Despite the above deviations from special relativity, a serious understanding of the studies herein considered requires the knowledge that the isotopies preserve by conception and technical realizations the abstract axioms of special relativity, and merely introduce broader realizations.
In fact, the Minkowski-Santilli isospacetime is locally isomorphic to the conventional space, the Lorentz-Santilli isosymmetry is locally isomorphic to the conventional isosymmetry, and so on.

Along these lines, Santilli has often indicated in his writing that the best way to honor the memory of Albert Einstein is to preserve his abstract axioms and introduce more adequate realizations, rather than adapting 20th century realizations of special relativity that are manifestly inapplicable and disproved by experimental evidence in any case (see general treatments [44])
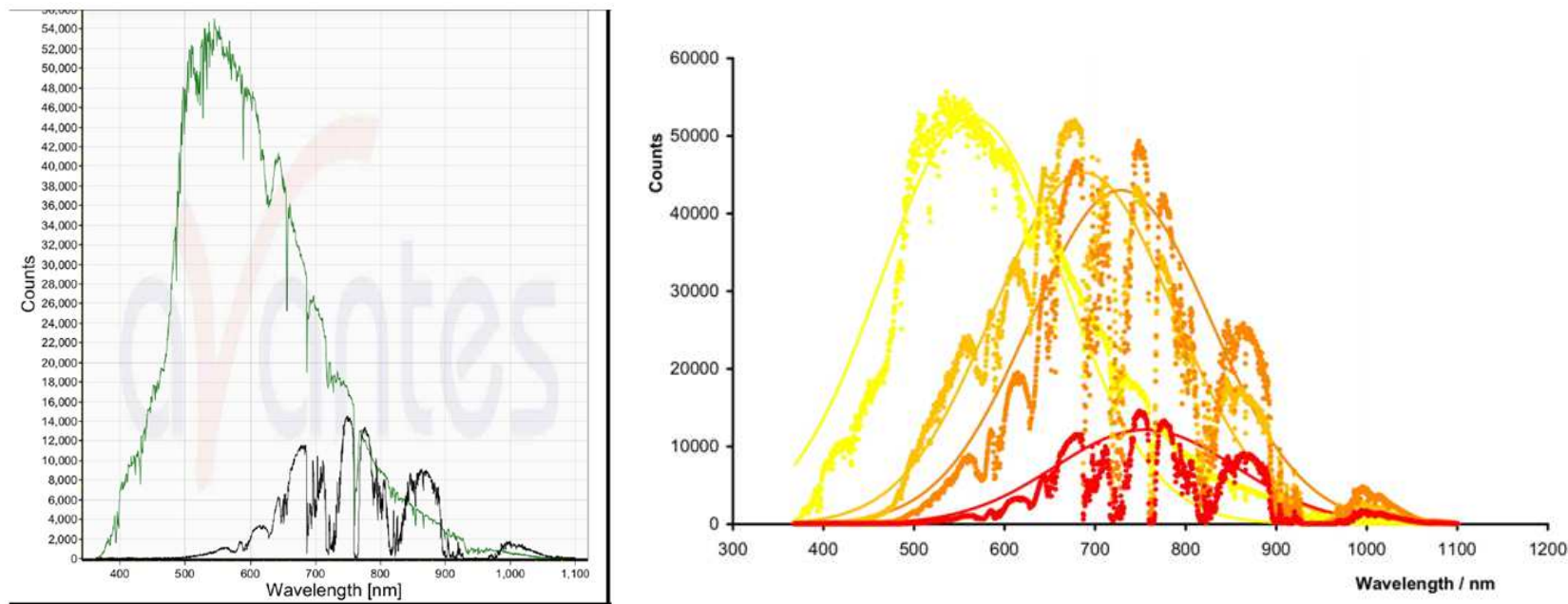

Figure 9. The first experimental confirmation that Sunlight experiences an IsoRedShift in the transition from the Zenith to the horizon obtained via a telescope focused on "direct" Sunlight and the use of a Yokogawa Wavelength analyzer capable of capturing the entire spectrum of Sunlight.

For independent mathematical studies on Santilli isomathematics and its physical applications we quote: Refs. 
$[45,46]$ for the direct universality of the Lorentz-Santilli isosymmetry;1 Refs., [28,29] on isonumbers; Refs. [49,50] on the Lie-Santilli isotheory; Refs. [51-53] on isogeometries; Ref. [54] on isomathematics; Refs. [55] for comprehensive mathematical studies i on Santilli isodifferential calculus and its vast applications; Ref. [56] for hypermathematics; Ref. [57] for a general independent review of mathematical, physical and chemical studies; and Ref. [58] provides detailed evidence for the dismissal of interpretations of measurements [37-53] in such a way to comply with the 20th century realization of special relativity.

\section{Lack of Expansion of the Universe and Related Conjectures}

As it is well known to historians, Albert Einstein, Edwin Hubble, Fritz Zwicky, Enrico Fermi, Louis de Broglie, and other famous scientists died without accepting the conjecture of the expansion of the universe because it implies a necessary return to the Middle Ages with Earth at the cementer of the Universe.

This implication is due to the fact that the experimental evidence on Hubble's law (1.1) establishes that the cosmological redshift is the same for all galaxies having the same distance $d$ from Earth, as generally admitted, "in all radial directions from Earth," as generally ignored.

The radial character of the cosmological redshift also applies to the conjectured acceleration of the expansion, and implies geometric inconsistencies illustrated in Fig. 10 that also invalidate the far reaching conjecture that space itself is expounding.

In fact, the latter conjecture was proposed for the intent of avoiding Earth at the center of the universe, which intent would have been achieved in the event the conjectured expansion of the universe had been uniform. Hence, Earth remains at the center of the universe even for the far reaching conjecture of the expansion of space itself due to the inherent acceleration of the expansion (Fig. 10).

In line with these objections, immediately following Hubble's discovery [1], F. Zwicky proposed in 1929 [59] the hypothesis that galactic light loses energy $E=\hbar \omega$ to the inter-0galactic medium during its long travel to reach Earth, by therefore experiencing a decrease of frequency $\omega$.

Zwicky hypothesis was dismissed, and continues to be generally dismissed on sole theoretical objections against the originally proposed scattering of light with inter-galactic gases, the expectation that galaxies could not be seen in the event galactic light would lose energy due to scattering, and other essentially conceptual objections.

About one century later, Santilli [37] has: constructed the novel isomathematics for the quantitative treatment of the propagation of light within media; identified the symmetry under which light can lose energy to physical media; confirmed Zwicky hypothesis via a series of experiments on Earth; and clarified that the mechanism for light losing energy to the inter-galactic medium is of atomic, rather than molecular character.

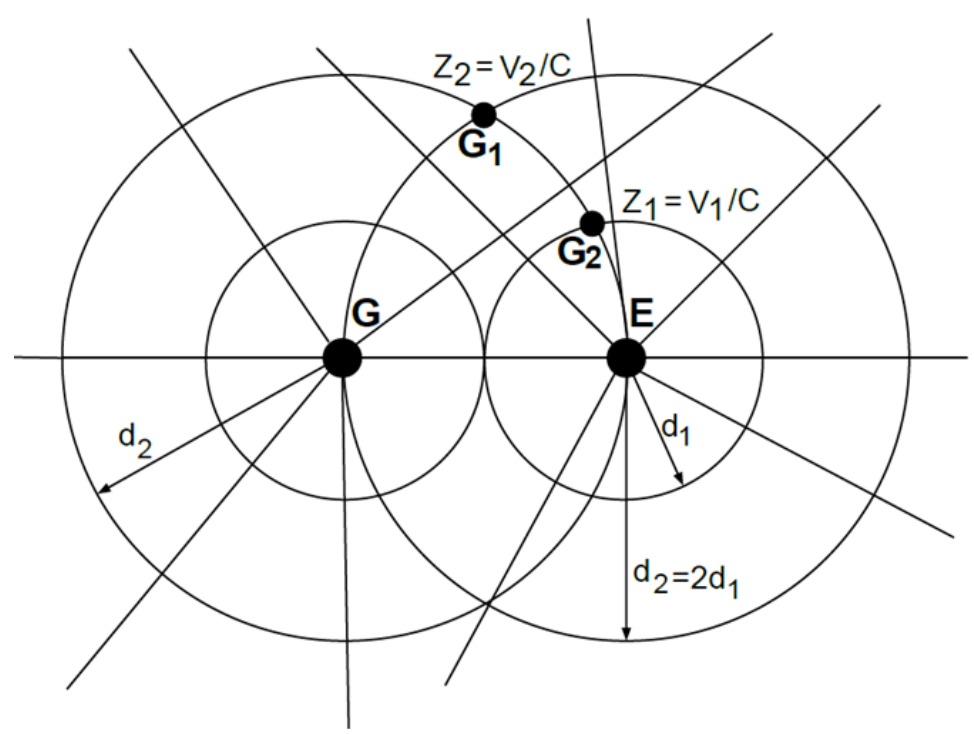

Figure 10. An illustration of the geometric inconsistencies of the conjecture of the expansion of the universe due to its inherent acceleration. According to conjecture (1.2), galaxy $G_{2}$ should have double speed as that of galaxy $G_{1}$ when seen from observer $E$, but the same galaxies have the same speed with respect to observer $G$, by therefore illustrating the reasons Einstein, Hubble, Zwicky, Fermi, de Broglie and other illustrious scientists died without accepting the expansion of the universe.

In view of these contributions, the loss of energy by light to the inter-galactic medium is now referred to as the Zwicky-Santilli effect [ [37-53].

The first clear experimental evidence that the universe is not expanding was achieved by Halton Arp [60] in the 1970s when he was at Harvard 's Astrophysics Laboratory via the discovery of quasars that are physically connected to a galaxy according to clear spectroscopic evidence, yet their cosmological redshifts are extremely different (top view of Fig. 11).

The validity of special relativity for the large scale structure of the universe would have implied that the quasars and the 
associated galaxy had to be separated billions of years ago and travel away from Earth at very different speeds, which conditions are disproved by astrophysical evidence.

Santilli provided in monographs [29] of 1991 a numerically exact and time invariant representation of the large difference in cosmological redshift of quasars and associated galaxies via the admission of astrophysical evidence according to which quasars have chromospheres that are dramatically denser than inner-galactic media. Therefore, light exits the quasar-galaxy pair and initiates its long travel to Earth already isoredshifted with large difference in cosmological redshift (bottom view of Fig. 11).

R. Mignani [61] confirmed in 1992 the validity of Santilli isoredshift interpretation of largely different cosmological redshifts for connected quasar-galaxies, and achieved the exact representation of astrophysical data of all Arp's pairs known at that time.

Systematic experimental evidence has been provided by Santilli [37-53] on the fact that the redness of the Sun at the horizon is indeed a redshift of the entire visible spectrum of light without any appreciable relative motion. Consequently, said redness is visual evidence of the lack of expansion of the universe (Figs. 8 and 9).

In particular, the redness of the Sun at the horizon provides a visual evidence not only on the isoredshift of Sunlight without relative motion, but also evidence of the increase of redness with the decease of the elevation, namely, with the increase of the distance $d$ covered by light in Earth's atmosphere (Fig. 8).

This constitutes visual evidence, confirmed by systematic measurements [37-52], on the lack of the expansion of the universe as well as on the lack of its acceleration.

Additional strong visual evidence has been provided in 2013 by astronaut Chris Hadfield who reported during his NPR interview [62] that his spaceship became "blood red" when seeing Sunrise, thus confirming that Sunlight passing through our atmosphere experiences a redshift without appreciable relative motion (Fig. 112, thus experiencing a isoredshift in full agreement with measurements [37-53].
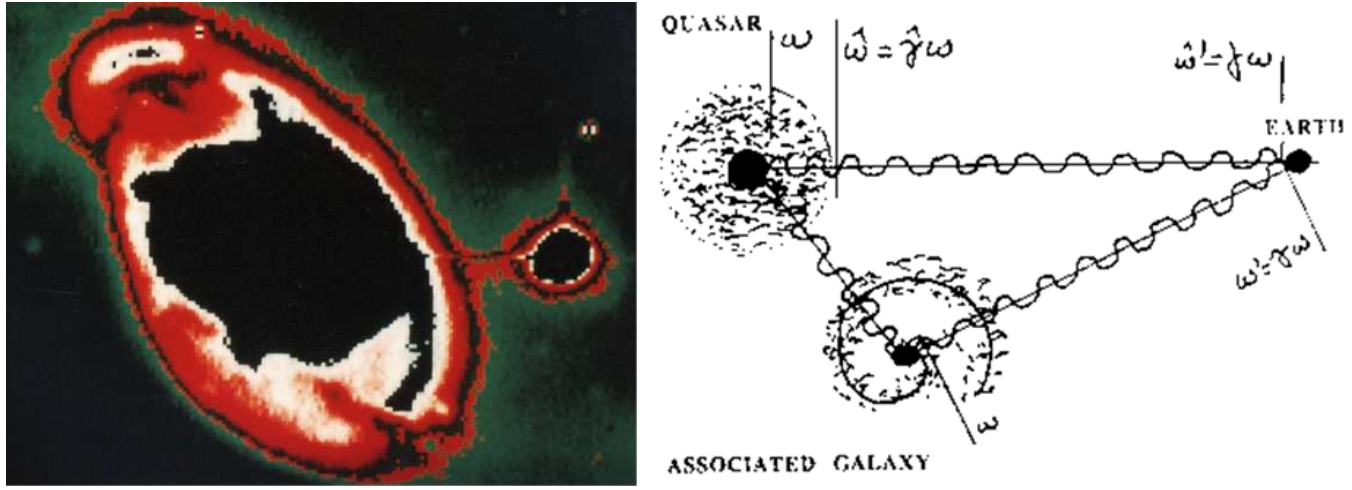

Figure11. Illustrations of the first experimental evidence on the inapplicability in astrophysics and cosmology of the $20^{\text {th }}$ century realization of special relativity achieved by A. Arp in the 1970s [60]. The left view illustrates a quasar which is physically connected to a galaxy according to clear gamma spectroscopic evidence. Their largely different cosmological redshift were explained by Santilli in 1991 [29] via dramatically different isoredshifts due to dramatically different media light has to propagate within prior to reaching intergalactic spaces.

When the Sun is seen millions of light years away, it also appears "blood red" without any expansion of the universe, because we have the replacement of Earth atmosphere with the inter-galactic medium. In both cases we have the experimentally verified [37-53] loss of energy $E=\hbar \omega$ by light to the medium with consequential decrease of the frequency.

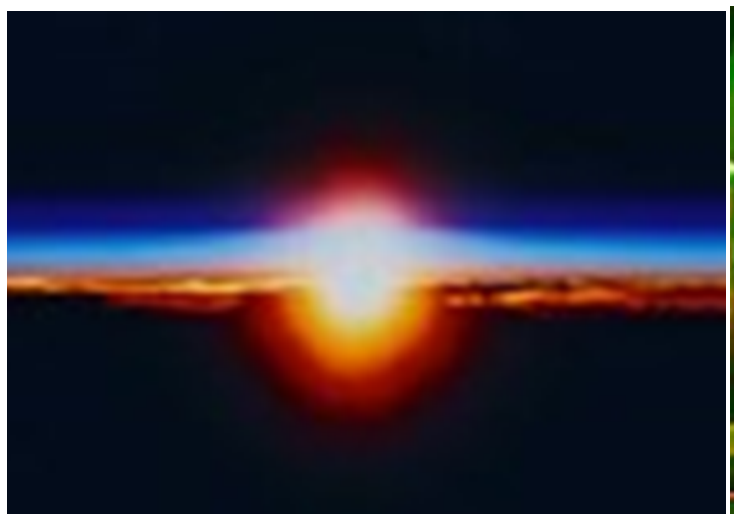

Note that, in this case, claims of contributions to the redshift of the Sun from Rayleigh or other scattering in Earth's atmosphere are readily dismissed since no scattering event can propagate light from the Sun to a spaceship in orbit. Only direct Sunlight can be seen from the spaceship. Its "red blood color" is then visual evidence of Santilli isoredshift.

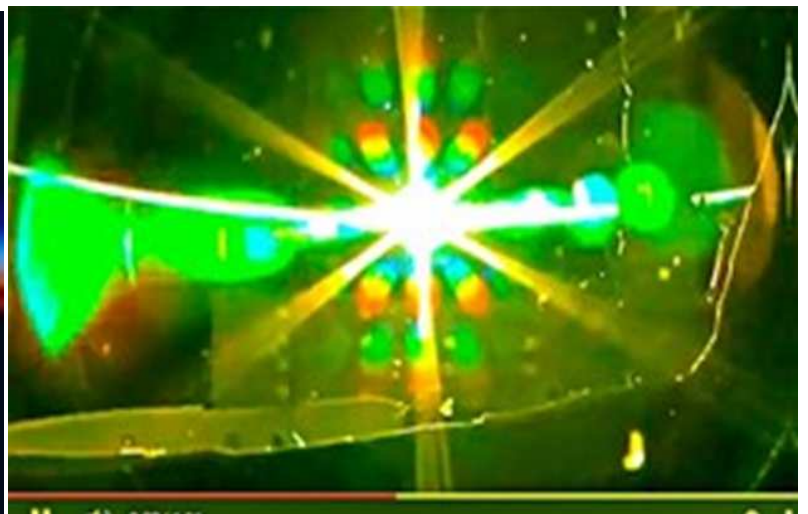

Figure 12. The "blood red" Sun (left view) spaceship interior (right view) as seen by astronaut Chris Hadfield in 2013 [62] provide additional visual evidence of Santilli isoredshift and of the lack of expansion of the universe. 
The frequently observed "blood red" Moon during Lunar eclipses (Fig. 13) provides an additional visual evidence of the lack of expansion of the universe because the Moon sees Sunlight redshifted while passing through Earth's atmosphere without appreciable relative motion, thus confirming Santilli isoredshift.

This is evidence that, light reaching us from far away galaxies is redshifted without the expansion of the universe due to the replacement of Earth's atmosphere with inter-galactic gases.

The above experimental and visual evidence on the lack of expansion of the universe also implies the elimination of" 1) The acceleration of the expansion; 2) The expansion of space itself'; 3) The big bang; 4) Cosmic inflation; and 5) The conjecture of dark energy (but it does not imply the conjecture of dark matter, as discussed in the next section).

Independently from the above evidence, the following comments should be kept in mind to conduct research in cosmology capable of passing the test of time.

Due top the inconsistencies of the far reaching conjecture that space itself is expanding 9Fig. 10), the acceptance of conjecture (1.2) implies the acceptance of entire galaxies at the edge of the universe traveling faster than the speed of light, by therefore contradicting the very reason for the expansion of the universe, namely, expand the validity of 20th century realization of special relativity to the entire large scale structure of the universe.

Independently from the above evidence, the big bang conjecture has been plagued by a number of generally unspoken or unaddressed insufficiencies or sheer inconsistencies, such as:

3.1) The big bang conjecture implies a necessary return to the Middle Ages with Earth at the Center of the universe as a necessary condition to verify Hubble's law (1.1) due to its radial character from Earth (Fig. 10).

3.2) By the very definition of "explosion" according to the Webster Dictionary, in the event the universe originated from a primordial explosion, our galactic environment should have no galaxies for 13.7 billions of light years or, in any case, the "debris" of the primordial explosion should have created a region of empty space around Earth.n This implication is disproved by astrophysical evidence on the distribution of galaxies in our environment;

3.3) Also according to the very definition of "explosion,"the speed of its "debris" (the galaxies) should decrease with the increase of the distance, as a result of which the big bang conjecture is incompatible with the very assumption of the expansion of the universe and its acceleration with the distance for which representation the conjecture was proposed.

3.4) The big bang conjecture provides no consistent model for the origin of the universe since the infinities that are inherent in a conjectured geometric singularity prevent any quantitative study of the real origin of the universe, not to mention the lack of addressing of what type of universe existed prior to the explosion.

3.5) It is generally claimed that the Cosmological Background Radiation is "experimental evidence" on the occurrence of the big bang. However, simple calculations show that the Cosmological Background Radiation should have been absorbed by galaxies and inter-galactic gases billions of years ago. Consequently, there exist no true experimental evidence to date on the occurrence of the conjectured big bang.

In reality, a quantitative representation of the Cosmological Background Radiation requires a continuous energy source for its persistence through time. Such a continuous source is indeed provided by Santilli's isoredshift representation of the cosmological redshift because the energy lost by galactic light to atoms of the inter-galactic medium is re-emitted via precisely weak electromagnetic waves via the de-excitation of the atoms under extremely low temperatures.

The conjecture of cosmic inflation is also afflicted by serious insufficiencies or sheer inconsistencies which are independent form the experimental evidence of its absence.

As an example, cosmic inflation is centrally dependent on Einstein general relativity, namely, on the curvature of space. However, the gravitational attraction and, therefore, the curvature of space, between galaxies has such an infinitesimal value to prevent physically meaningful calculation on gravitational inflation.
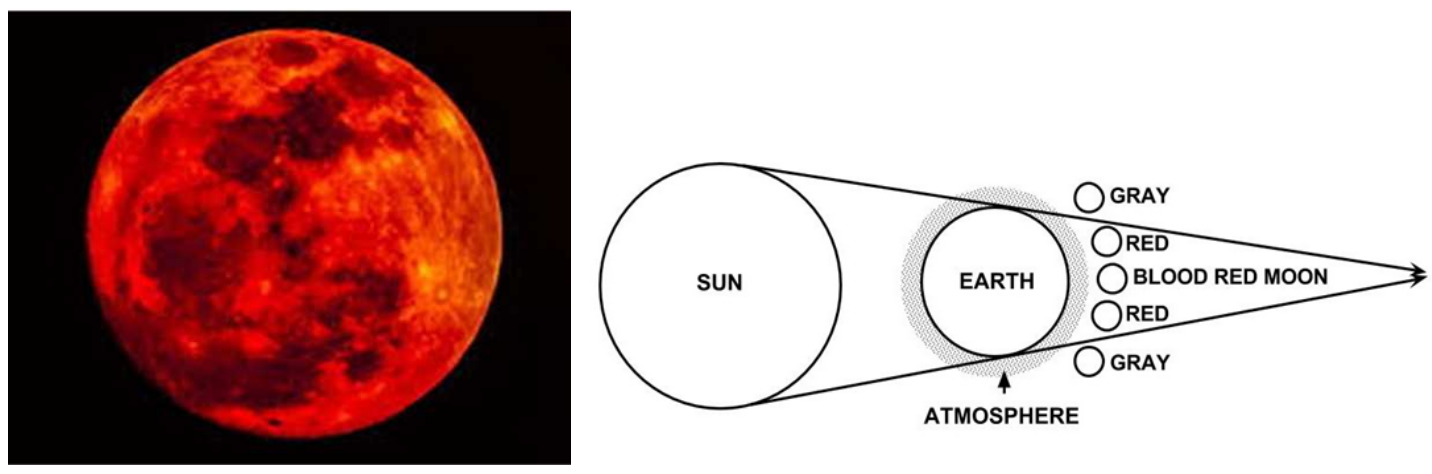

Figure 13. The "blood red" Moon during a Lunar eclipse (left view) is yet an additional visual evidence of Santilli isoredshift because the redshift occurs for Sunlight passing though Earth's atmosphere without any appreciable relative motion. This provides an additional confirmation that the Sun is seen redshifted at millions of light years distance without any expansion of the universe due to the replacement of Earth's atmosphere with the inter-galactic medium. 
Finally, the conjecture of dark energy is perhaps the most afflicted by controversies, insufficiencies or inconsistencies among all above indicated cosmological conjectures.

As a matter of fact, according to Einstein general relativity, in the event the universe had been composes in a numerically predominant way of any form of energy, the universe should contract, and definitely should not expand. This is due to the generally forgotten fact that, according to Einstein, the source of gravitation is energy, and not mass.

The conjecture of dark energy did succeed to deviate attention from the inapplicability of special relativity for the large scale structure of the universe, but no consistent quantitative reconciliation of the conjectured expansion of the universe with the prediction of contraction by established theories has been achieved to date on and, additionally, no model expected to pass the test of time has been provided to the author's best knowledge on how energy could cause gravitational repulsion.

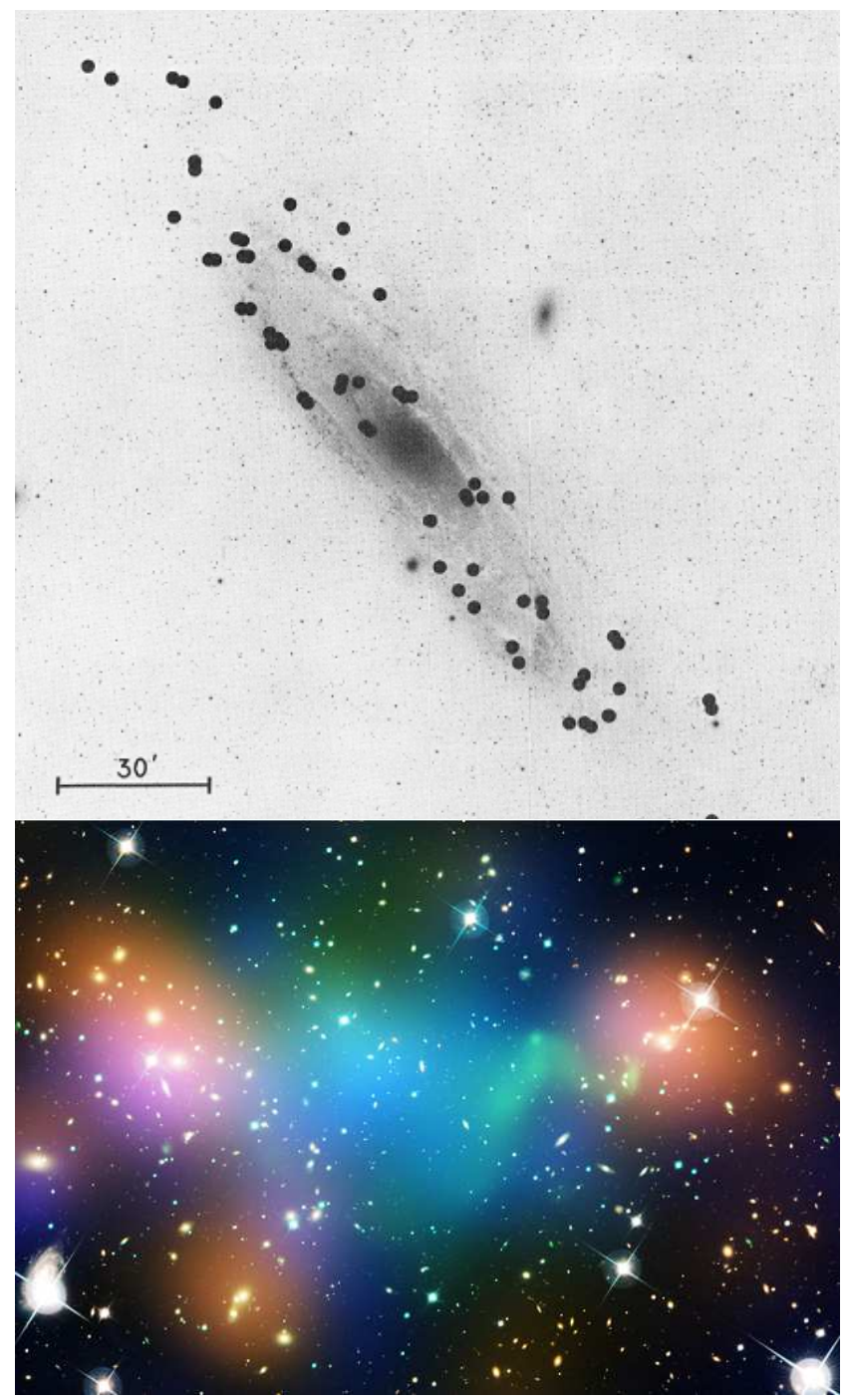

Figure 14. Visual evidence from Ref. [8] (top view) of physical media inside galaxies, and evidence of physical media permeating clusters of galaxies from NASA records (bottom view).

\section{Representation of Anomalous Galactic Frequency Shifts Via Isoshifts without Dark Matter}

\subsection{Basic Assumptions}

Our central contention is that all galaxies are indeed filled up with matter, but rather than being invisible and hypothetical, galaxies are filled up with an actual, physical, measurable medium, hereon called inner-galactic medium, constituted by gases, elementary particles, electromagnetic radiations, and force fields, which medium ranges from high temperatures and densities in the vicinity of galactic centers to very low temperatures and densities for peripheral stars (see Fig 14).

The admission of the astrophysical evidence on the existence of the inner-galactic medium renders simply inevitable the study of galactic star redshifts via Santilli isoredshifts and isoblueshifts that establish via experiments on Earth the inapplicability (and not the violation) for galactic star dynamics of the 20th century realization of special relativity in general, and of Doppler's shift law $(1,5)$ in particular, as studied in Refs. [20-59], and reviewed in Sections 2 and 3.

It should be stressed that the indicated inapplicability refers, specifically, to the "20th century realization of special relativity" because Einstein's abstract axioms of special relativity remain exactly valid, since they merely require a broader realization permitted by Santilli isomathematics, for which very purpose isomathematics was initiated and mostly developed for by the author.

In this section we present, apparently for the first time, a numerically exact and time invariant representation of galactic star redshifts via Santilli isoredshift and isoblueshift based on the admission of inner-galactic medium without any use of the conjecture of dark matter, under the following:

ASSUMPTION 4.1: Quantitative elaborations of data for galactic star dynamics are conducted via Santilli isomathematics for an axiomatically correct and time invariant geometrization of the density, temperature, inhomogeneity, anisotropy and other characteristics of inner-galactic media.

ASSUMPTION 4.2: Newton gravitation is assumed to be exactly valid for the dynamics of galactic stars. Consequently, rotational motions are computed under the validity of Keplerian laws, except for second order corrections, such as the slow down of orbital motions of galactic stars caused by the resistance during their motion within a physical medium.

ASSUMPTION 4.3: The anomalous redshift of individual galactic stars with respect to the cosmological redshift of the galaxy as a whole is due to the experimentally verified Santilli isoredshifts or isoblueshift caused by the propagation of starlight within the inner-galactic medium. 


\subsection{Representation of Anomalous Frequency Shifts of Galactic Stars}

In Section 1 we have shown that rotational speeds cannot produce a measurable frequency shift due to the essentially null value of the ratio $v_{s} / c$. Consequently, in this section we focus our attention on the only available experimental evidence on galactic star dynamics, which is their redshift [8].

To achieve the desired numerical representation, we re-scale in Fig. 15 the theoretically assumed rotational values of Fig. 1 in term of the anomalous redshift $z_{s}$ of individual galactic stars in such a way that, when summed up with the cosmological redshift $z_{g}$ the galaxy as a whole, we have the actual, physical, measured, total redshift (1.7).

For this purpose, we note that Assumptions 4.1, .4.2 and 4.3 imply the geometrization of the inner-galactic medium via the Minkowski-Santilli isospacetime (2.6), the invariance under the Lorentz-Santilli isosymmetry (2.7), and Santilli isoshift law (2.11) which we rewrite in the form

$$
z=\frac{\omega_{e}}{\omega_{o}}-1=z_{g}+z_{s} \approx \pm F_{g}(t, r, v, \rho, \tau, \omega, \ldots) d_{g} \pm F_{s}(t, r, v, \rho, \tau, \omega, \ldots) d_{s},
$$

where: $z$ is the total, solely measured, frequency shift of light from individual galactic stars;

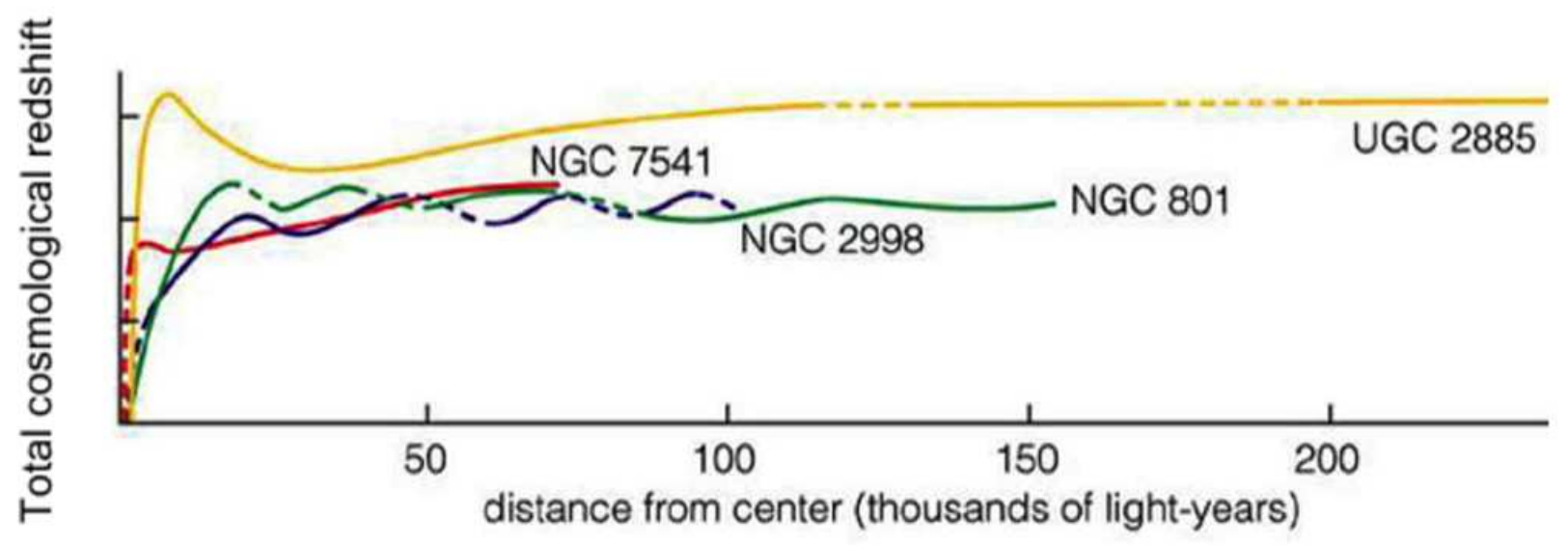

Figure 15. The diagram represents the reconstruction of the experimental values of Ref. [8], those in term of anomalous redshifts of individual galactic stars, rather than in terms of rotational motions as done in Ref. [8] (Fig. 1), since the latter were theoretically assumed in Ref. [8] under Assumptions 1.1,1.2 and 1.3.

$z_{g}$ is the cosmological frequency shift of a galaxy as a whole; $z_{s}$ is the anomalous frequency shift of individual galactic stars, namely, its deviation from the value $z_{g} ; F_{g}$ is the characteristic function representing the characteristics of the inter-galactic medium; $d_{g}$ is the distance traveled by light within the inter-galactic medium to reach Earth; $F_{s}$ is the characteristic function representing the characteristic of the inner-galactic medium; $d_{s}$ is the distance traveled by light within the inner-galactic medium until exiting the galaxy; positive signs denoted isoredshifts; negative signs denotes isoblueshifts; and all characteristic quantities (as well as distances) are assumed to be positive-definite according to rule (2.2).

It is easy to see that frequency shift law (4.1), denoted isoshift in the literature, provides a numerically exact representation of the anomalous redshift of individual galactic stars as represented in the diagram of Fig. 15. In particular, such a representation is invariant over time because isolaw (4.1) is uniquely and unambiguously characterized by the Lorentz-Santilli isosymmetry (2.7).

As a first example, consider galaxy NGC8001 of Fig. 15 and assume that the redshift at maximal distance from the galactic center is the cosmological redshift of the entire galaxy.
This assumption is supported by the fact that, for peripheral galactic stars, the value $d_{g}$ (and, therefore, the redshift $z_{g}$ )is ignorable with respect to $d_{c}$ (and, therefore, with respect to $\left.z_{c}\right)$.

With the decrease of the distance of a star from the galactic center, we see from Fig. 15 a decrease of the total redshift $z$ for galaxy NGC8001 beginning at approximately 50 light years from the galactic center. This is evidence that the inner-galactic frequency shift is an isoblueshift according to the isolaw

$$
z=z_{g}+z_{s} \approx+F_{g} d_{g}-F_{s} d_{s}
$$

thus implying the values

$$
F_{s} d_{s} \leq F_{g} d_{g}
$$

where the equality can at best occur (for galaxy NGC801) at close proximity of the galactic center.

In turn, the above representation implies that, beginning from about 50 light years of distance from the galactic center, the inner-galactic medium begins to have an average temperature bigger than $120^{\circ} \mathrm{F}$ (Section 2).

Said temperature then continues to increase with the 
decrease of the distance from the galactic center, jointly with an evident increase of the density, depending on local characteristics of composition, inhomogeneity, other factors, and other physical data.

A numerically exact and time invariant representation of the experimental data on the anomalous redshift of individual stars of galaxy NGC8001 as presented in Fig. 15 is then provided by a mere fit of the characteristic function $F_{s}(t, r, v, \rho, \mu, \tau, \omega, \ldots)$ to the related curve of Fig. 15. This fit can be obtained nowadays via mere computerization, e. g., as function of the local temperature $\tau$ and density $\mu$ and, as such, its explicit form (which fit varies from galaxy to galaxy) is scheduled for detailed study in a subsequent paper.

In essence, with the approaching to the galactic center, light emitted by galactic stars passes through the chromospheres of other stars and other high temperature gases before exiting the galaxy, thus acquiring energy $E=\hbar \omega$ in the process, with consequential increase of the frequency $\omega$ (Fig. 3) as experimentally established for isoblueshifts on Earth (Section 2).

However, the value of the isoblueshift remains smaller than the cosmological redshift of the galaxy as a whole, thus resulting in the decrease of the cosmological redshift, without turning the cosmological redshift into a blueshift for the galaxy considered.

By contrast, it is known that certain galaxies do have a cosmological blueshift which is interpreted as being due to (relativistic) motion of the entire galaxy toward us. Santilli isoblueshifts show that, in this case, the inner-galactic medium is so hot and dense that the galactic star isoblueshift becomes bigger than the cosmological redshift, thus resulting in a total isoblueshift $z$ of Eq. (4.1).

Essentially the same quantitative and time invariant representation holds for the behavior of the anomalous redshift for galaxies NGC 2998m NGC 7541 and many other galaxies not considered here for brevity. In all these cases, local fluctuations of anomalous individual star redshifts with respect to the cosmological redshift of the galaxy as a whole can be equally represents via isolaw (4.1) under realization (4.2) and (4.3).

Consider now the galaxy UGC 3885 of Fig. 15. In this case, the long horizontal value of the redshift is evidence of the thin and low temperature character of inner-galactic medium. The increase of the redshift beginning at about 20 light year distance from the galactic center is evidence of the existence of a cold inter-galactic medium with increasing density.

In this case, rather than isolaws (4.2) and (4.3), we have the isolaw

$$
z=z_{g}+z_{s} \approx F_{g} d_{g}+F_{s} d_{s}
$$

with the prediction of galaxies for which we have the total isoshift

$$
\begin{gathered}
z=z_{g}+z_{s} \approx F_{g} d_{g}-F_{s} d_{s}, \\
F_{g} d_{g} \leq \mid F_{s} d_{s},
\end{gathered}
$$

in which case galactic light measured on Earth is blueshifted.

It should be stressed that the above quantitative and time invariant representations are solely valid outside galactic centers because inspection of the galactic star redshift of Fig. $1 / 5$ clearly indicates that, in the vicinity of galactic centers, the total frequency shift can indeed became an isoblueshift, as expected from the high temperature and density in the vicinity of black holes, thus confirming again Santilli isoshifts [37-53].

\subsection{Representation of Anomalous Frequency Shifts of Galactic Clusters}

As it is well known, there exist clusters of galaxies (Fig. 14) at close distance from each other with anomalous frequency shifts similar to those for galactic stars. In this case, the theoretical assumption of rotational speeds of galaxies from the measured frequency ships is so big to activate the virial theorem according to which a minimum value of a rotating mass, called virial mass, is needed for the clusters to remain together (see, e.g., the historical account [63] and the recent account [64] with vast references quoted therein).

Various calculations indicate that the mass of the galaxies is at least one tenth of the virial mass, under which assumption galaxies are expected to leave the clusters and disperse in inter-galactic spaces contrary to astrophysical evidence. In order to verify the virial theorem, a generally accepted, although directly unverifiable conjecture is that clusters of galaxies are filled up with such an amount of dark matter to verify the virial theorem [loc. cit.].

Regrettably, this additional use of the conjecture of dark matter is afflicted by the same insufficiencies as those for galactic stars (see Insufficiencies 1.1 to 1.e). In fact, from the very large value of galactic distances from Earth compared to inner-galactic distances in a cluster, rotation in a plane perpendicular to the distance from Earth provide no appreciable contributions to frequency shifts, and rotations in a plane at an angle with the distance from Earth provide opposite contributions with respect to the rotation center, both conditions being disproved by astrophysical evidence.

Additionally, from the proportionality of possible contributions to frequency shifts to the ratio $v / c$, rotational speeds of galaxies should be relativistic to achieve appreciable values, in which case it would not be possible to see individual galaxies in a given cluster since at relativistic rotations we could only see blobs of light. This inevitable consequence is also disproved by astrophysical observations according to which galaxies in a given cluster have been individually visible and their locate has remained unchanged over one century of records.

As it was the case for the anomalous frequency shifts of individual galactic stars, astrophysical evidence illustrated by Fig. 14 establishes the existence of a dense medium inside galactic clusters, which medium invalidates the exact applicability of Doppler's frequency shift (1.5) according to experiments on Earth [37-53]. Consequently, we have the invalidation of rotational speeds crucially based on Doppler's shift law. Moreover, said medium activates Santilli isoredshifts (isoblueshifts) according to which anomalous 
frequency shifts are due to release (absorption) of energy to (from) the medium at low (high) temperature without any activation of the virial theorem.

The admission of a real, physical, measurable medium within galactic clusters then allows a numerical exact and time invariant representation of anomalous frequency shifts via isolaw (4.1). As a matter of fact, anomalous frequency shifts provide important information on the characteristics of the medium filling up galactic clusters, with numerical values of temperature, densities, distributions, and other data. Since these value evidently vary from cluster to cluster, their detailed study will be presented in the above indicated subsequent paper.

In summary, without any need to conjecture that galaxies are filled up with hypothetical, invisible and undetectable matters whose conjectured effect on individual stars does not represent the experimental data of Fig. 1, it appears that the experimental data on the total redshift of galactic stars, when compared to the cosmological redshift of galaxies, provides valuable data on the actual physical characteristics of the inner-galactic medium, such as temperature, density, inhomogeneity, anisotropy, etc.

We should finally note that all aspects of the representation of anomalous galactic star isoshifts presented in this section can be subjected to additional experimental verifications on Earth, besides the experimental verifications of Santilli isoredshift and isoblueshift reviewed in Sections 2 and 3 [37-53].

As one example among several possible proposed tests, the isoblueshifts of galactic light, when passing through a hot gaseous medium can be verified by measuring the frequency of galactic light before and while passing though the Sun chromosphere or some hot planetary atmosphere.

\section{Conclusions}

The conjecture of dark energy is only one of a rather large number of directly unverifiable conjectures to extend the applicability of the $20^{\text {th }}$ century realization of special relativity, from its original conditions of applicability and experimental verifications (point particles and electromagnetic waves moving in empty space represented via inertial reference systems), to all possible conditions existing in the large scale structure of the universe.

In fact, besides the conjecture of dark matter, we have the additional conjectures of the expansion of the universe, the acceleration of the expansion, the big bang, the cosmic inflation, the dark energy, the neutralino, the sterile neutrinos, the WIMPs (weakly interacting massive particles not seen in particle laboratories), and other conjectures under consideration, none of which conjectures being directly verifiable on Earth, and each conjecture being generally aimed at resolving insufficiencies of preceding conjectures.

Albert Einstein, Edwin Hubble, Fritz Zwicky, Enrico Fermi, Luis de Broglie and other famous scientists died without accepting the conjecture of the expansion of the universe and related conjectures because, as indicated in Section 3, they imply a necessary return to the Middle Ages with Earth at the center of the universe due to the radial character of both, he expansion of the universe and its acceleration.

Far from intending to venture personal opinions, the mathematical, theoretical and experimental works conducted by the author in cosmology [20-53] has provided experimental evidence on Earth dismissing all the indicated cosmological conjectures for the intent of honoring the memory of the 20th century founding fathers of our scientific knowledge.

We can readily state that studies on the application of 20th century realization of special relativity to all possible conditions existing in the large scale structures of the universe should continue. However, an effective way to honor the memory of Albert Einstein is that of maintaining the axioms of special relativity at the abstract level, and introducing their broader realization via isorelativity $[23,29,44,65]$ for interior physical conditions beyond those of the original conception and experimental verification of special relativity.

\section{References}

[1] E. Hubble, Proceedings of the National Academy of Sciences of the United States of America, 15, 168 (1929).

[2] H. A. Lorentz, Amst. Proc. 6, 809 (1904).

[3] H.Poincaré, Compte Rendues, Paris 140. 1504 (1905).

[4] H.Minkowski, Nachr. Ges. Wiss. Gottingen 43 (1908).

[5] A.Einstein, Ann. Phys. 17, 891 (1905).

[6] P. J. E. Peebles and B. Ratra, The Cosmological Constant and Dark Energy, Rev Mod. Phys. 758559 (2003).

[7] M. Moriconi, "Special theory of relativity through the Doppler effect" Eur. J. Phys. 271409 (2006).

[8] V. Rubin and Ford, W. Kent, Jr. "Rotation of the Andromeda Nebula from a Spectroscopic Survey of Emission Regions". The Astrophysical Journal 159: 379403 (1970).

[9] A. Drukier, K. Freese, and D. Spergel, D. (1986). "Detecting Cold Dark Matter Candidates". Physical Review D 33 (12): 34953508(1986).

[10] Trimble, V. (1987). "Existence and nature of dark matter in the universe". Annual Review of Astronomy and Astrophysics 25: 425472. Bibcode:1987ARAA..25..425T (1987).

[11] Drukier, A.; Freese, K. and Spergel, D. (1986). "Detecting Cold Dark Matter Candidates". Physical Review D 33 (12): 34953508 .

[12] W. Atwood, et al., "The large area telescope on the Fermi Gamma-ray Space Telescope Mission". Astrophysical Journal 697 (2): $1071 Đ 1102$ (2006).

[13] J. Albert, J. et al., (2008). "Upper Limit for ??Ray Emission above $140 \mathrm{GeV}$ from the Dwarf Spheroidal Galaxy Draco". The Astrophysical Journal 679: 428 (2008).

[14] R. Bernabei etal., (2008). "First results from DAMA/LIBRA and the combined results with DAMA/NaI". Eur. Phys. J. C 56 (3): 333355 (2008). 
[15] O. Adriani, O. et al., "An anomalous positron abundance in cosmic rays with energies 1.5100 ? GeV". Nature 458 (7238): 607609 (2009).

[16] C. Weniger, "A Tentative Gamma-Ray Line from Dark Matter Annihilation at the Fermi Large Area Telescope". Journal of Cosmology and Astroparticle Physics 2012 (8): 7 (2012);

[17] S. Archambault et al., "Constraints on low-mass WIMP interactions on 19F from PICASSO", Phys. Lett. B, 711, 153 (2012).

[18] R. Agnese et al. (SuperCDMS Collaboration), "Search for Low-Mass Weakly Interacting Massive Particles with SuperCDMS",Phys. Rev. Lett. 112, 241302 (2014).

[19] M. Schumann, "Dark Matter 2013", Brazilian Journal of Physics Volume 44, Issue 5, pp 483-493 (2014).

[20] R. M. Santilli, Foundation of Theoretical Mechanics, Volume I (1978) [1a], and Volume II (1982) [1b], Springer-Verlag, Heidelberg, http://www.santilli-foundation.org/docs/Santilli-209.pdf, http://www.santilli-foundation.org/docs/santilli-69.pdf

[21] R. M. Santilli, "Isonumbers and Genonumbers of Dimensions 1, 2, 4, 8, their Isoduals and Pseudoduals, and "Hidden Numbers" of Dimension 3, 5, 6, 7," Algebras, Groups and Geometries Vol. 10, 273 (1993), http://www.santilli-foundation.org/docs/Santilli-34.pdf

[22] R. M. Santilli, "Nonlocal-Integral Isotopies of Differential Calculus, Mechanics and Geometries," in Isotopies of Contemporary Mathematical Structures, P. Vetro Editor, Rendiconti Circolo Matematico Palermo, Suppl. Vol. 42, 7-82 (1996), http://www.santilli-foundation.org/docs/Santilli-37.pdf

[23] R. M. Santilli, Elements of Hadronic Mechanics, Vol. I (1995) [13a], Vol. II 1995) [13b] Academy of Sciences, Kiev, available in free pdf downloads from http://www.santilli-foundation.org/docs/Santilli-300.pdf, http://www.santilli-foundation.org/docs/Santilli-301.pdf

[24] R. M. Santilli, "Lie-admissible invariant representation of irreversibility for matter and antimatter at the classical and operator levels," Nuovo Cimento B 121, 443 (2006), available in free pdf download from the link http://www.santilli-foundation.org/docs/Lie-admiss-NCB-I.pd f

[25] R. M. Santilli, "Iso-, geno-, hyper-relativities for matter and their isoduals for antimatter, and their novel applications in physics, chemistry and biology,"Journal of Dynamical Systems and Geometric Theories, Vol. 1, 121-193 (2003).

[26] B. Davvaz, R. M. Santilli and T. Vougiouklis Mathematical prediction of Ying's twin universes American Journal of Modern Physics, Vol. 4, pp 5-9 (2015), $\mathrm{http} / / / \mathrm{www}$. santilli-foundation.org/docs/ying-twing-univ.pdf

[27] R. M. Santilli, "Lie-isotopic Lifting of Special Relativity for Extended Deformable Particles," Lettere Nuovo Cimento 37, 545 http://www.santilli-foundation.org/docs/Santilli-50.pdf

(1983),

[28] R. M. Santilli, "Lie-isotopic Lifting of Unitary Symmetries and of Wigner's Theorem for Extended and Deformable Particles," Lettere Nuovo Cimento Vol. 38, 509 (1983), http://www.santilli-foundation.org/docs/Santilli-51.pdf

[29] R. M. Santilli, Isotopic Generalizations of Galilei and Einstein
Relativities, Vols. I [3a] and II [3b] (1991), Ukraine Academy of Sciences, http://www.santilli-foundation.org/docs/Santilli-01.pdf, http://www.santilli-foundation.org/docs/Santilli-61.pdf

[30] R. M. Santilli, "Isotopies of Lie Symmetries," I (basic theory) and II (isotopies of the rotational symmetry), Hadronic J. Vol. 8, 36 http://www.santilli-foundation.org/docs/santilli-65.pdf (1985),

[31] R. M. Santilli, "Isotopic Lifting of the SU(2) Symmetry with Applications to Nuclear Physics," JINR rapid Comm. Vol. 6. 24-38 (1993), http://www.santilli-foundation.org/docs/Santilli-19.pdf

[32] R. M. Santilli, "Nonlinear, Nonlocal and Noncanonical Isotopies of the Poincaré Symmetry," Journal of the Moscow Phys. Soc. Vol. 3, 255 (1993), http://www.santilli-foundation.org/docs/Santilli-40.pdf

[33] R. M. Santilli, "Recent theoretical and experimental evidence on the synthesis of the neutron," Communication of the JINR, Dubna, Russia, No. E4-93-252 (1993).

[34] R. M. Santilli, "Isominkowskian Geometry for the Gravitational Treatment of Matter and its Isodual for Antimatter," Intern. J. Modern Phys. D 7, 351 (1998), http://www.santilli-foundation.org/docs/Santilli-35.pdf

[35] R. M. Santilli, "Isorepresentation of the Lie-isotopic SU(2) Algebra with Application to Nuclear Physics and Local Realism," Acta Applicandae Mathematicae Vol. 50, 177 (1998), http://www.santilli-foundation.org/docs/Santilli-27.pdf

[36] R. M. Santilli, "Direct universality of isospecial relativity for photons with arbitrary speeds" in Photons: Old problems in Light of New Ideas, V. V. Dvoeglazov Editor Nova Science (2000), http://www.santilli-foundation.org/docs/Santilli-24.pdf

[37] R. M. Santilli, "Experimental Verifications of IsoRedShift with Possible Absence of Universe Expansion, Big Bang, Dark Matter, and Dark Energy," The Open Astronomy Journal 3, 124 (2010),

http://www.santilli-foundation.org/docs/Santilli-isoredshift.pdf

[38] R. M. Santilli, "Experimental Verification of IsoRedShift and its Cosmological Implications," contributed paper to the Proceedings of the International Conference on Numerical Analysis and Applied Mathematics, Rhodes, Greece, September 19-25, 2010, T. E. Simos, Editor, American Institute of Physics Conference Proceedings Vol. 1281, pp. 882-885 (2010),

http://www.santilli-foundation.org/docs/Isoredshift-Letter.pdf

[39] R. M. Santilli, G. West and g. Amato, "Experimental Confirmation of the IsoRedShift at Sun at Sunset and Sunrise with Consequential Absence of Universe Expansion and Related Conjectures, " Journal of Computational Methods in Sciences and Engineering, Vol. 12, pages 165-188 (2012), http://www.santilli-foundation.org/docs/Confirmation-sun-IRS .pdf

[40] G. West and G. Amato, "Experimental Confirmation of Santilli's IsoRedShift and IsoBlueShift," contributed paper to the Proceedings of the San Marino Workshop on Astrophysics and Cosmology for Matter and Antimatter, Republic of San Marino, September 5 to 9, 2011, in press (2012), http://www.santilli-foundation.org/docs/Confirmation-IRS-IB S.pdf 
[41] H. Ahmar, G. Amato, J. V. Kadeisvili, J. Manuel, G. West, and O. Zogorodnia, "Additional experimental confirmations of Santilli's IsoRedShift and the consequential lack of expansion of the universe,"Journal of Computational Methods in Sciences and Engineering, Vol. 13, page 321 (2013), $\mathrm{http} / / / w w w . s a n t i l l i-f o u n d a t i o n . o r g /$ docs/IRS-confirmations-21 2.pdf

[42] S J. Dhoble, "Cosmological implications of Santilli IRS and IBR," to appear (2014) http://www.santilli-foundation.org/docs/irs-ibs-2014.pdf ,

[43] R. Anderson, et al, Editors, San Marino Workshop on Astrophysics and Cosmology for Matter and Antimatter, $\begin{array}{lllll}\text { September } & 5 & \text { to } & 9 \text {, }\end{array}$ http://www.workshops-hadronic-mechanics.org main lectures available in free view or download from the link http://www.world-lecture-series.org/san-marino-2011, and lectures from subsequent meetings in the links http://www.world-lecture-series.org/level-v, $\mathrm{http}: / / \mathrm{www}$. world-lecture-series.org/san-marino-2011, http://www.world-lecture-series.org/kos-2012, http://www.world-lecture-series.org/invited-2014-keynote-lect ures

[44] R. M. Santilli, Hadronic Mathematics, Mechanics and Chemistry,, Vol. I [18a], II [18b], III [18c], IV [18d] and [18e], International Academic Press, http://www.i-b-r.org/Hadronic-Mechanics.htm

[45] J. V. Kadeisvili, "Direct universality of the Lorentz-Poincare'-Santilli isosymmetry for extended-deformable particles, arbitrary speeds of light and all possible spacetimes" in Photons: Old problems in Light of New Ideas, V. V. Dvoeglazov Editor Nova Science (2000, available as free download from http://www.santilli-foundation.org/docs/Santilli-25.pdf

[46] A. K. Aringazin and K. M. Aringazin, "Universality of Santilli's iso-Minkowskian geometry" in Frontiers of Fundamental Physics, M. Barone and F. Selleri, Editors Plenum http://www.santilli-foundation.org/docs/Santilli-29.pdf (1995),

[47] Chun-Xuan Jiang, Foundations of Santilli Isonumber Theory, International Academic Press (2001), http://www.i-b-r.org/docs/jiang.pdf

[48] Christian Corda An Introduction to Santilli Iso-Numbers AIP Conf. Proc. 1558, 685 (2013), http://www.santilli-foundation.org/docs/Isonumbers.pdf

[49] D. S. Sourlas and Gr. T. Tsagas, Mathematical Foundation of the Lie-Santilli Theory, Ukraine Academy of Sciences (1993), http://www.santilli-foundation.org/docs/santilli-70.pdf

[50] J. V. Kadeisvili, "An introduction to the Lie-Santilli isotopic theory," Mathematical Methods in Applied Sciences, 19, 1349 (1996), available in the website http://www.santilli-foundation.org/docs/Santilli-30.pdf

[51] A. K. Aringazin, A. Jannussis, F. Lopez, M. Nishioka and B. Veljanosky, Santilli's Lie-Isotopic Generalization of Galilei and Einstein Relativities, Kostakaris Publishers, Athens, Greece (1991), available as free download from http://www.santilli-foundation.org/docs/Santilli-108.pdf
[52] Raul M. Falcon Ganfornina and Juan Nunez Valdes, Fundamentos de la Isoteoria de Lie-Santilli, International Academic Press (2001), http://www.i-b-r.org/docs/spanish.pdf English edition in press at Springer

[53] J. V. Kadeisvili, "Foundations of the Lie-Santilli Isotheory," in "Isotopies of Contemporary Mathematical Structures," P. Vetro Editor, Rendiconti Circolo Matematico Palermo, Suppl. Vol. 42, 7-82 http://www.santilli-foundation.org/docs/Santilli-37.pdf

(1996),

[54] A. S. Muktibodh, Foundations of Isomathematics AIP Conference Proceedings 1558, p. 707 (2013), http://www.santilli-foundation.org/docs/isomathematics-AIP.p df

[55] S. Georgiev, Foundations of the IsoDifferential calculus, Volumes I, II, III, IV, V, VI and VII, Nova Science Publishers, (2014 on)

[56] B. Davvaz, Hyperings Theory and Applications, International Academic Press (2007), http://www.santilli-foundation.org/docs/Davvaz.pdf

[57] I. Gandzha and J. Kadeisvili, New Sciences for a New Era" Mathematical, Physical and Chemical Discoveries of Ruggero Maria Santilli, Sankata Printing Press, Nepal (2011), available in free pdf download from the link http://www.santilli-foundation.org/santilli-scientific-discoverie s.html

[58] R. Cox, "Comments on I. Gandzha's Paper: "Solar Redshift Calculation by the Rayleigh Scattering Mechanism," Hadronic J. 37, 33 (2013), http://www.scientificethics.org/Criticisms-Gandzha.pdf

[59] F. Zwicky, "On the Red Shift of Spectral Lines through Interstellar Space," Proceedings of the National Academy of Sciences of the United States of America, 15, 773 (1929) http://www.pnas.org/cgi/reprintframed/15/10/773

[60] H. Arp. Quasars Redshift and Controversies. Interstellar Media, Berkeley (1987).

[61] R. Mignani. Physics Essay 1992; 5, 531, available in pdf download from the link http://www.santilli-foundation.org/docs/Santilli-31.pdf

[62] C. Hadfield, NPR Interview, October 20, (2014) http://www.prweb.com/releases/2013/12/prweb11435701.htm

[63] F. Zwicky, "On the Masses of Nebulae and of Clusters of Nebulae", Astrophys. J. 86, 217 (1937).

[64] A, Vikhlinin, et al., "Chandra Sample of Nearby Relaxed Galaxy Clusters: Mass, Gas Fraction, and MassĐTemperature Relation". The Astrophysical Journal 640 (2): 691709 (2006).

[65] R. M. Santilli, "Isolinear, isoinvariant isorelativity," lectures http://www.world-lecture-series.org/isorelativity-2014-i http://www.world-lecture-series.org/isorelativity-2014-ii http://www.world-lecture-series.org/isorelativity-2014-iii 